\title{
STABILITY THEOREMS FOR SOME FUNCTIONAL EQUATIONS
}

\author{
BY \\ R. C. MACCAMY( $\left.{ }^{1}\right)$ AND J. S. W. WONG
}

Abstract. Functional-differential equations of the form

$$
\dot{u}(t)=-\int_{0}^{t} A(t-\tau) g(u(\tau)) d \tau+f(t, u(t))
$$

are considered. Here $u(t)$ is to be an element of a Hilbert space $\mathscr{H}, A(t)$ a family of bounded symmetric operators on $\mathscr{H}$ and $g$ an operator with domain in $\mathscr{H} . g$ may be unbounded. $A$ is called strongly positive if there exists a semigroup exp $S t$, where $S$ is symmetric and $(S \xi, \xi) \leqq-m\|\xi\|^{2}, m>0$, such that $A^{*}=A-\exp S t$ is positive, that is,

$$
\int_{0}^{T}\left(v(t), \int_{0}^{t} A^{*}(t-\tau) v(\tau)\right) d \tau \geqq 0,
$$

for all smooth $v(t)$. It is shown that if $A$ is strongly positive, and $g$ and $f$ are suitably restricted, then any solution which is weakly bounded and uniformly continuous must tend weakly to zero. Examples are given of both ordinary and partial differentialfunctional equations.

1. Introduction. This paper concerns a class of nonlinear functional-differential equations. A prototype for this class is the equation

$$
\dot{u}(t)=-\int_{0}^{t} a(t-\tau) g(u(\tau)) d \tau
$$

This equation has been the subject of a great deal of work, primarily that of Levin and Nohel. The main goal is to obtain conditions on $a$ and $g$ which guarantee that all solutions tend to zero as $t$ tends to infinity. The first result in this direction [9] asserts that asymptotic stability holds if $u g(u)$ is positive when $u \neq 0$ and $a$ is completely monotone, that is,

$$
a^{\prime}(t) \not \equiv 0, \quad(-1)^{k} a^{(k)}(t) \geqq 0, \quad k=0,1,2, \ldots
$$

This result was improved in [8] where it is shown that (1.2) need hold only for $k=0,1,2,3$. More recently Hannsgen [6] has shown that (1.2) need hold only for

Received by the editors May 11, 1970 and, in revised form, June 4, 1971.

AMS 1969 subject classifications. Primary 3930.

Key words and phrases. Functional-differential equation, Gårding's inequality, Laplace transform, partial differential-functional equation, positivity, strong ellipticity, strong positivity, symmetric, weak boundedness, weak convergence, weak stability, weak uniform continuity.

(1) This research was supported by NSF Grant GP-11945.

Copyright (C) 1972, American Mathematical Society 
$k=0,1,2$. At the same time it has been shown that one can obtain some stability results for the perturbed equation

$$
\dot{u}(t)=-\int_{0}^{t} a(t-\tau) g(u(\tau)) d \tau+f(t, u(t)) .
$$

All of the results of Hannsgen, Levin and Nohel were obtained through the use of Lyapunov functions. There was however one paper, by Halanay [4], which attempted to treat (1.1) by different methods. Halanay asserted asymptotic stability of the zero solution of (1.1) under assumption (1.2), $k=0,1,2$. Halanay's proof is incorrect at several places. However, it seems to us that the proof contains an idea of great interest and importance. It is this idea which is developed in the present paper and we proceed to describe it here in a general way.

One can show that if $g$ satisfies certain conditions and $a$ is a positive kernel, that is, for any $h \in C[0, \infty)$,

$$
q(T ; h)=\int_{0}^{T} h(t) \int_{0}^{t} a(t-\tau) h(\tau) d \tau d t \geqq 0,
$$

for all $T$, then all solutions of (1.1) remain bounded for all $t$. It is known that $a$ is a positive kernel if its Laplace transform $\hat{a}(s)$ satisfies the condition

$$
\operatorname{Re} \hat{a}(s)>0 \quad \text { in } \operatorname{Re} s>0 .
$$

It can be shown (see $\$ 4$ ) that (1.2) for $k=0,1,2$ implies (1.5) and hence stability of the zero solution of (1.1). Halanay's idea was that, in fact, (1.2) for $k=0,1,2$ implies something stronger than (1.4) for $a$ and hence yields asymptotic stability. He attempted to show that if (1.2) holds for $k=0,1,2$ then for some positive $\varepsilon$ and $\alpha$ the kernel $\tilde{a}(t)=a(t)-\varepsilon e^{-\alpha t}$ is still positive. This has the effect of sharpening (1.4) to

$$
\int_{0}^{T} h(t) \int_{0}^{t} a(t-\tau) h(\tau) d \tau d t \geqq \frac{\varepsilon}{2} \zeta^{2}(T)+\alpha \varepsilon \int_{0}^{T} \zeta^{2}(t) d t
$$

where

$$
\zeta(t)=\int_{0}^{t} e^{-\alpha(t-\tau)} h(\tau) d \tau .
$$

It turns out that the rather curious estimate (1.6) is sufficient to yield asymptotic stability of solutions of (1.1). Halanay's proof that $\varepsilon$ and $\alpha$ can be found is incorrect. However he had what we believe is the essential idea, namely that what is necessary is roughly that (1.5) be sharpened to

$$
\operatorname{Re} \hat{a}(s)>0 \quad \text { on } \operatorname{Re} s=0, \quad s \neq 0 .
$$

In this paper we both clarify and extend Halanay's idea. When it is properly phrased it becomes clear that it can be extended to systems of equations and even to certain partial differential equations. Thus we phrase the whole theory on a 
Hilbert space $\mathscr{H}$ allowing $g$ to be an unbounded operator but requiring $a$ to be bounded and linear. The essential idea is to extend the inequality (1.6), which we term strong positivity. In the general setting the role of $e^{-\alpha t}$ is taken over by a semigroup whose infinitesimal generator is negative definite.

We apply the notion of strong positivity only in very special cases. However the theory seems to us to be of sufficient interest on its own to warrant a more general discussion on Hilbert spaces and we present this in sections two and four. The discussion centers around the Laplace transform of the kernel $a$ and we show that (1.7) is indeed the key requirement. We show that (1.2) for $k=0,1,2$ is sufficient for strong positivity on finite dimensional spaces but not in general. On the other hand we consider the case $\boldsymbol{R}^{1}$ in detail and show that (1.2) is in no sense necessary. In fact we show that there exist oscillatory kernels, for example $e^{-t} \cos t$, which are strongly positive and also that there are strongly positive kernels which become infinite at $t=0$. The main tool for applications, which we obtain in $\S 2$, is what we call the weak stability principle. This states that if $h(t)$ is weakly bounded and weakly uniformly continuous in $t \geqq 0$ and $q(T ; h)$ is bounded for all $T$ then $h$ must tend weakly to zero as $t$ tends to infinity.

In $\S 3$ we present a discussion of the generalized equations (1.1) and (1.3). We distinguish between positive and strongly positive kernels, showing that, roughly speaking, positivity yields stability while strong positivity yields asymptotic stability. Our main result is of a conditional nature and has the following general form. If $a$ is strongly positive and $u$ is a solution of (1.3) such that $g(u)$ is weakly bounded and weakly uniformly continuous then $g(u)$ tends weakly to zero as t tends to infinity.

There are of course a number of technical hypotheses which are required and we discuss these in detail in $\$ 3$. The interesting feature, though, is the "weak-weak" character of the result. In finite dimensional spaces this aspect ceases to be of importance hence does not appear in Halanay's work. For partial differential equations, however, it becomes very important for it is the weak boundedness and continuity which is most easily verified. Moreover weak convergence of $g(u)$ in one space, together with standard embedding theorems, yields strong convergence of $u$ to zero in a different space. It is thus interesting that it is the "weak-weak" results which come out naturally from the theory. The corresponding "strongstrong" results (in obvious terminology) require stronger hypotheses on $a$ but at the same time are not as interesting.

In $\$ 5$ we give extensive and concrete applications of the theory to the equation (1.3) in $\boldsymbol{R}^{1}$. We obtain most of the known results as well as some new ones which help to clarify the roles of the various special hypotheses on $g$ and $f$ which occur in earlier papers. Our work on strongly positive kernels shows that (1.2), $k=0,1,2$, are only one set of hypotheses and that the results may hold for oscillatory kernels or kernels which are infinite at zero. We have included in $\$ 5$ a heuristic discussion of the linear version of (1.3) and we then proceed to obtain generalizations of the 
results to nonlinear equations. We call attention in particular to Theorem 5.6 in which we show that if $a \in L_{1}$ and $f(t, u)=f_{\infty}+h(t), h \in L_{1}$, then solutions of (1.3) need not tend to zero.

For equations in $\boldsymbol{R}^{1}$ we are able to verify the necessary boundedness and uniform continuity of solutions from the equations themselves. It will be clear that most of the results in $\boldsymbol{R}^{1}$ carry over directly to equations in $\boldsymbol{R}^{n}$ although we do not discuss these explicitly. For partial differential-functional equations the situation is not so simple. To illustrate this we present a brief discussion of such equations in $\$ 6$. We study primarily the linear equation

$$
u_{t}(x, t)=-\int_{0}^{t} a(t-\tau) L u(x, \tau) d \tau+f(x, t)
$$

where $L$ is a strongly elliptic differential operator and $a$ is a strongly positive kernel in $\boldsymbol{R}^{\mathbf{1}}$. We show how the general theory of this paper connects to (1.8) through standard results in partial differential equation theory and thus we obtain asymptotic stability results for (1.8). These are related to some recent work of Dafermos [1], in which he extends the ideas of Levin and Nohel to equations on Hilbert spaces.

It is only in the linear case that we are able to carry through the theory completely. We present, in $\S 6$, a simple example of a nonlinear equation for which we can prove the weak boundedness but not the weak uniform continuity.

2. Weak stability principle. Let $\mathscr{H}$ be a Hilbert space, and $C_{\mathscr{H}}[0, \infty)$ be the space of all continuous functions on $[0, \infty)$ with values in $\mathscr{H} . \tilde{A}=\{A(t): t \in[0, \infty)\}$ is a strongly continuous one parameter family of bounded linear operators on $\mathscr{H}$. For fixed $T>0$, we define the functional $Q_{\tilde{A}}[v ; T]$ on $C_{\mathscr{H}}[0, \infty)$ by

$$
Q_{\tilde{A}}[v ; T]=\int_{0}^{T}\left(v(t), \int_{0}^{t} A(t-\tau) v(\tau) d \tau\right) d t,
$$

where $(\cdot, \cdot)$ denotes the inner product on $\mathscr{H}$.

We say that the one parameter family $\tilde{A}$ defines a positive kernel if

$$
Q_{\tilde{A}}[v ; T] \geqq 0 \quad \text { for all } v \in C_{\mathscr{H}}[0, \infty) \text { and } T \geqq 0 .
$$

It is easy to see that if $A(t)=c I$ for all $t \in[0, \infty)$ where $c$ is a nonnegative real constant and $I$ denotes the identity operator on $\mathscr{H}$, then

$$
Q_{c I}[v ; T]=\frac{c}{2}\left\|\int_{0}^{T} v(\tau) d \tau\right\|^{2} \geqq 0 \text { for all } T>0,
$$

and thus $c I$ defines a positive kernel.

We now introduce the notion of strongly positive kernels. Let $S$ be a symmetric linear operator with domain dense in $\mathscr{H}$ and such that

$$
(S \xi, \xi) \geqq \sigma\|\xi\|^{2}, \quad \sigma>0, \xi \in \mathscr{D}_{s} .
$$


Consider the uniformly continuous one parameter semigroup $\tilde{S}$ generated by $S$, namely, $\tilde{S}=\left\{k e^{-S t}: t \in[0, \infty)\right\}, k>0$. Consider now the functional $Q_{\tilde{s}}[v ; T]$ associated with $\tilde{S}$, that is,

$$
Q_{\tilde{s}}[v ; T]=\int_{0}^{T}\left(v(t), k \int_{0}^{t} e^{-S(t-\tau)} v(\tau) d \tau\right) d t .
$$

We set

$$
s[v](t)=\int_{0}^{T} e^{-S(t-\tau)} v(\tau) d \tau
$$

and observe that

$$
(d / d t) s[v](t)=v(t)-S(s[v](t)) .
$$

We form the inner product of (2.6) with $s[v](t)$ and obtain

$$
\frac{1}{2}(d / d t)\|s[v](t)\|^{2}=(v(t), s[v](t))-(S s[v](t), s[v](t)) .
$$

For fixed $T>0$, we integrate equation (2.7) from 0 to $T$ and obtain, by (2.4),

$$
Q_{\tilde{s}}[v ; T] \geqq \frac{1}{2} k\|s[v](T)\|^{2}+k \sigma \int_{0}^{T}\|s[v](t)\|^{2} d t .
$$

We use the condition (2.8) to define the concept of a strongly positive kernel. We say that $\tilde{A}$ defines a strongly positive kernel if there exists a symmetric linear operator $S$, satisfying (2.4), and $k>0$ such that if $\tilde{S}=k e^{-S t}$, then, for all $T>0$,

$$
Q_{\tilde{A}}[v ; T] \geqq Q_{\tilde{S}}[v ; T] \text { for all } v \in C_{\mathscr{H}}[0, \infty) \text {. }
$$

Clearly, any one parameter family $\tilde{A}$ defining a strongly positive kernel also defines a positive kernel.

We call an element $v(t)$ in $C_{\mathscr{H}}[0, \infty)$ weakly stable if, for each $w \in \mathscr{H}$, the function $(v(t), w)$ is bounded and uniformly continuous in $t$. In other words, $v(t) \in C_{\mathscr{H}}[0, \infty)$ is weakly stable if $v(t)$ is weakly bounded and weakly uniformly continuous. The following result forms the basis of our present study. We term it the weak stability principle.

THEOREM (2.1). Suppose that $\tilde{A}$ is strongly positive and $v(t)$ is a weakly stable element in $C_{\mathscr{H}}[0, \infty)$. If $Q_{\tilde{A}}[v ; T]$ is bounded for all $T$, then $v(t)$ converges weakly to zero.

Proof. By hypothesis there exists a symmetric $S$, satisfying (2.4), such that if

$$
s[v](t)=\int_{0}^{t} e^{-S(t-\tau)} v(\tau) d \tau
$$

then we have

$$
Q_{\tilde{A}}[v ; T] \geqq c_{1}\|s[v](T)\|^{2}+c_{2} \int_{0}^{T}\|s[v](t)\|^{2} d t
$$


where $c_{1}, c_{2}>0$. For any $w \in \mathscr{D}_{s}$, we have, from (2.6),

$$
(d / d t)(s[v](t), w)=(v(t), w)-(S s[v](t), w) .
$$

We observe that (2.11) and the assumption that $Q_{\tilde{A}}[v ; T]$ is bounded for all $T>0$ imply that

$$
|(S s[v](t), w)| \leqq\|s[v](t)\|\|S w\|,
$$

which is bounded for all $t \geqq 0$. Since $(v(t), w)$ is bounded, by hypothesis, it follows that the function $\varphi(t)=(s[v](t), w)$ has a bounded derivative, hence is uniformly continuous. Furthermore it belongs to $L_{2}[0, \infty)$ since

$$
\int_{0}^{T}|(s[v](t), w)|^{2} d t \leqq\|w\|^{2} \int_{0}^{T}\|s[v](t)\|^{2} d t
$$

It follows that $\lim _{t \rightarrow \infty} \varphi(t)=0$. The uniform continuity of $\varphi(t)$ implies that of $(S s[v](t), w)=(s[v](t), S w)$, therefore $\varphi^{\prime}(t)$ is uniformly continuous on account of (2.12) and the weak uniform continuity of $v(t)$. It is then an immediate consequence of the mean value theorem that $\varphi^{\prime}(t)$ tends to zero (see $\left.\S 5\right)$. Since $(S s[v](t), w)$ $=(s[v](t), S w)$ tends to zero, (2.12) implies that

$$
\lim _{t \rightarrow \infty}(v(t), w)=0,
$$

completing the proof.

A natural question to ask, in light of Theorem (2.1), is whether strong boundedness and uniform continuity of $g(u)$ imply strong convergence to zero. It turns out that this is not true without further assumptions. We hope to return to this at a later time.

3. Functional-differential equations. We consider functional-differential equations of the form

$$
\dot{u}(t)=-\int_{0}^{t} A(t-\tau) g(u(\tau)) d \tau+f(t, u(t)), \quad t \geqq 0,
$$

on a Hilbert space $\mathscr{H}$. Here $\tilde{A}=\{A(t): t \geqq 0\}$ is a strongly continuous, one parameter family of bounded linear operators on $\mathscr{H}, g$ is a transformation with domain $\mathscr{D}_{g} \subseteq \mathscr{H}$, and $f(t, v)$ is a mapping from $[0, \infty) \times \mathscr{D}_{g}$ into $\mathscr{H}$. By a solution of (3.1), we mean a map $u:[0, \infty) \rightarrow \mathscr{H}$ which satisfies the following conditions:

(i) $u$ is strongly continuous on $[0, \infty)$;

(ii) $u(t) \in \mathscr{D}_{g}$ for all $t \in(0, \infty)$;

(iii) $u$ is strongly differentiable on $(0, \infty)$;

(iv) $u$ satisfies $(3.1)$ on $(0, \infty)$.

We leave aside questions of existence and uniqueness of solutions of (3.1) although these are closely related to our stability results, especially on finite 
dimensional spaces. For convenience, we refer to (3.1) as the perturbed equation and to its special cases

$$
\dot{u}(t)=-\int_{0}^{t} A(t-\tau) g(u(\tau)) d \tau+f(t), \quad t \geqq 0,
$$

and

$$
\dot{u}(t)=-\int_{0}^{t} A(t-\tau) g(u(\tau)) d \tau
$$

as the unperturbed and homogeneous equations respectively.

We establish the connection between (3.1) and the ideas of the preceding section by forming the inner product of (3.1) and $g(u(t))$ and integrating from 0 to $T$. The result is the following:

$$
\int_{0}^{T}(\dot{u}(t), g(u(t))) d t+Q_{\tilde{A}}[g(u) ; T]=\int_{0}^{T}(f(t, u(t)), g(u(t))) d t .
$$

Our goal is to find conditions on $f$ and $g$ such that the positivity of $\tilde{A}$ will guarantee, by (3.1), the boundedness of $Q_{\tilde{A}}[g(u) ; T]$. Then if we impose strong positivity on $\tilde{A}$, we can infer from Theorem $(2.1)$ that if $g(u)$ is weakly stable it must tend to zero weakly. The verification of weak stability of solutions of (3.1) is carried out, for the most part, in the special cases discussed in detail in $\$ \S 5$ and 6. However, we do present some general results applicable to (3.1).

Our basic hypothesis on $g$ and $f$ are as follows:

$\left(\mathrm{G}_{0}\right)$ There exists a functional $G(v)$ defined on $\mathscr{D}_{g}$ which satisfies

(i) $\inf _{v \in \mathscr{D}_{g}} G(v)>-\infty$,

(ii) $G(v)$ is Fréchet differentiable on $\mathscr{D}_{g}$ and $\operatorname{grad} G(v)=g(v)$ for all $v \in \mathscr{D}_{g}$, i.e. $G(v+h)=G(v)+(g(v), h)+o(\|h\|)$, as $\|h\| \rightarrow 0$.

$\left(\mathrm{F}_{0}\right)$ There exists a function $\alpha \in L_{1}[0, \infty)$ such that

$$
|(f(t, v), g(v))| \leqq \alpha(t)(1+G(v)),
$$

for all $t \geqq 0$ and for all $v \in \mathscr{D}_{g}$. The functional $G$ is as postulated in $\left(\mathrm{G}_{0}\right)$.

Our basic boundedness and stability result is the following.

Theorem (3.1). Suppose that $\left(\mathrm{G}_{0}\right)$ and $\left(\mathrm{F}_{0}\right)$ hold, and that $u(t)$ is a solution to (3.1).

(i) If $\tilde{A}$ defines a positive kernel, then $|G(u(T))|$ and $Q_{\tilde{A}}[g(u) ; T]$ are bounded for all $T \geqq 0$.

(ii) If $\tilde{A}$ defines a strongly positive kernel, and if $g(u)$ is a weakly stable element in $C_{\mathscr{H}}[0, \infty)$, then $g(u)$ tends weakly to zero.

Proof. Using the positivity of $\tilde{A}$, we obtain from (3.4) the following estimate:

$$
\int_{0}^{T}(\dot{u}(t), g(u(t))) d t \leqq \int_{0}^{T}(f(t, u(t)), g(u(t))) d t .
$$


Assumptions $\left(\mathrm{G}_{0}\right),\left(\mathrm{F}_{0}\right)$ and an application of the chain rule together imply $\left({ }^{2}\right)$

$$
G(u(T))-G(u(0))=\int_{0}^{T} \frac{d}{d t} G(u(t)) d t \leqq \int_{0}^{T} \alpha(t)(1+G(u(t))) d t,
$$

from which it follows that

$$
|G(u(T))| \leqq\left(|G(u(0))|+\int_{0}^{\infty} \alpha(t) d t\right) \int_{0}^{T} \alpha(t) d t<\infty .
$$

Substituting (3.6) into (3.4), we obtain

$$
Q_{\tilde{A}}[g(u) ; T] \leqq|G(u(T))|+|G(u(0))| \int_{0}^{T} \alpha(t) d t+\sup _{t \geqq 0}|G(u(t))| \int_{0}^{\infty} \alpha(t) d t,
$$

proving the boundedness of $Q_{\tilde{A}}[g(u) ; T]$. The conclusion (ii) of the theorem then follows from Theorem (2.1).

REMARK (3.1). The boundedness of $G$ need not of course imply the boundedness of either $g(u)$ or $u$. If we impose on $g$ the additional hypothesis

$$
G(v) \rightarrow \infty \text { as }\|v\| \rightarrow \infty,
$$

then (3.6) will imply that $\|u(t)\|$ is bounded. If in addition $g$ is assumed to be a bounded mapping, then it will follow that $g(u)$ is also bounded (hence, of course, weakly bounded). Thus, for example, on finite dimensional spaces, $\left(G_{1}\right)$ and the continuity of $g$ will yield the boundedness of $g(u)$. The boundedness of $g(u)$ together with additional assumptions on $\tilde{A}$ and $f$ will yield boundedness results for $\dot{u}(t)$ and $\ddot{u}(t)$, namely

Corollary (3.1). Suppose that $\left(\mathrm{G}_{0}\right),\left(\mathrm{F}_{0}\right),\left(\mathrm{G}_{1}\right)$ hold and that $\tilde{A}$ defines a positive kernel. If in addition $g$ is a bounded mapping, then for every solution $u(t)$ of (3.2), we have

(i) $\|A(t)\| \in L_{1}[0, \infty),\|f(t)\| \leqq B_{1} \Rightarrow\|\dot{u}(t)\| \leqq M_{1}$ for all $t \geqq 0$;

(ii) $\|\dot{A}(t)\| \in L_{1}[0, \infty),\|\dot{f}(t)\| \leqq B_{2} \Rightarrow\|\ddot{u}(t)\| \leqq M_{2}$.

Proof. From Theorem (3.1) and the discussion just given in Remark (3.1), we know that $\|g(u(t))\|$ is bounded for all $t \geqq 0$, say by $B_{0}$. Returning to equation (3.1), we have

$$
\begin{aligned}
\|\dot{u}(t)\| & \leqq B_{0} \int_{0}^{t}\|A(t-\tau)\| d \tau+\|f(t)\| \\
& \leqq B_{0} \int_{0}^{t}\|A(\sigma)\| d \sigma+B_{1},
\end{aligned}
$$

$\left({ }^{2}\right)$ The necessary condition on $G$ is that $(d / d t) G(u)(t)=(g(u(t)), \dot{u})$. We are showing here that $\left(G_{0}\right)$ implies this. In $\S 6$ we will not have $\left(G_{0}\right)$ but the differentiation result will be verified directly. 
proving (i). To prove (ii), we differentiate (3.1) to obtain

$$
\ddot{u}(t)=-A(0) g(u(t))-\int_{0}^{t} \dot{A}(t-\tau) g(u(\tau)) d \tau+\dot{f}(t),
$$

which gives

$$
\|\ddot{u}(t)\| \leqq B_{0}\|A(0)\|+B_{0} \int_{0}^{t}\|\dot{A}(t-\tau)\| d \tau+\|\dot{f}(t)\| .
$$

This establishes the result.

REMARK (3.2). For the homogeneous equation (3.3), assumption $\left(F_{0}\right)$ is automatically satisfied. For the unperturbed and nonhomogeneous equation (3.2), $\left(\mathrm{F}_{0}\right)$ will be satisfied if we assume

$\left(\mathrm{F}_{1}\right)\|f(t)\| \in L_{1}[0, \infty)$, and

$\left(\mathrm{G}_{2}\right)\|g(v)\| \leqq k(1+|G(v)|)$ for all $v \in \mathscr{D}_{g}$.

ReMARK (3.3). When $g(u)$ is a linear symmetric transformation, $g(u)=L u$, we can satisfy (ii) of $\left(\mathrm{G}_{0}\right)$ by taking $G(u)=\frac{1}{2}(u, L u)$. Condition (i) will then be satisfied if $L$ is positive and $\left(\mathrm{G}_{1}\right)$ will be satisfied if the smallest eigenvalue of $L$ is positive. If $L$ is in addition bounded, then the last condition is also sufficient for $\left(G_{2}\right)$ to hold. This will be the case in finite dimensional spaces. In $\boldsymbol{R}^{1}$, we can satisfy (ii) of $\left(\mathrm{G}_{0}\right)$ by taking $G(u)=\int_{0}^{u} g(\xi) d \xi$ and (i) of $\left(\mathrm{G}_{0}\right)$ will be satisfied if, for example, $g \in C(-\infty, \infty)$ and $u g(u) \geqq 0$ for $|u|$ large. For a general discussion concerning the existence of the functional $G(v)$, see Vainberg [15].

Theorem (3.1) is capable of an extension which is important in the applications. We are here concerned with the condition $\left(\mathrm{F}_{0}\right)$ which in general rules out a constant perturbing term. We thus replace $\left(\mathrm{F}_{0}\right)$ by the more general condition

$\left(\tilde{\mathrm{F}}_{0}\right) f(t, u)=f_{\infty}+f_{1}(t, u)$, where $f_{\infty} \in \mathscr{H}$ and $f_{1}$ is a mapping from $[0, \infty) \times \mathscr{H}$ into $\mathscr{H}$ which satisfies $\left(\mathrm{F}_{0}\right)$.

We shall show later (in $\S 5$ ) that when $f_{\infty} \neq 0$, one cannot, in general, expect asymptotic stability even if $\mathscr{H}=\boldsymbol{R}^{1}$. On the other hand, if $A(t)$ also contains a constant term, then we can still obtain asymptotic stability as we show now.

THEOREM (3.2). Suppose that $\left(\mathrm{G}_{0}\right)$ and $\left(\tilde{\mathrm{F}}_{0}\right)$ hold, and that $u(t)$ is a solution of (3.1). Assume that the operator limit $A(\infty)$ exists and defines a positive definite symmetric operator and let $\tilde{A}_{0}=A-A(\infty)$. Then the following hold:

(i) If $\tilde{A}_{0}$ defines a positive kernel, then $|G(u(T))|$ and $Q_{\tilde{A}}[g(u) ; T]$ are bounded for all $T \geqq 0$.

(ii) If $\tilde{A}_{0}$ defines a strongly positive kernel, then $g(u)$ weakly stable in $C_{\mathscr{H}}[0, \infty)$ $\Rightarrow g(u(t))$ tends weakly to zero.

Proof. The proof involves a process of "completing the square" and was used by Levin and Nohel in the one dimensional case. We rewrite (3.1) in the form

$$
\dot{u}(t)=-A(\infty) \int_{0}^{t} g(u(\tau)) d \tau-\int_{0}^{t} A_{0}(t-\tau) g(u(\tau)) d \tau+f(t, u(t)) .
$$


Now form the inner product of (3.8) with $g(u(t))$ and integrate from 0 to $T$. With $(\xi, A(\infty) \xi) \geqq a_{0}\|\xi\|^{2}$ for all $\xi \in \mathscr{H}$, one obtains,

$$
\begin{aligned}
G(u(T))-G(u(0)) & +Q_{\tilde{A}_{0}}[g(u) ; T] \\
& \leqq-a_{0}\left\|\int_{0}^{T} g(u(t)) d t\right\|^{2}+\left(f_{\infty}, \int_{0}^{T} g(u(t)) d t\right)+\int_{0}^{T} \alpha(t)(1+G(u(t))) d t \\
& \leqq \frac{1}{4 a_{0}}\left\|f_{\infty}\right\|^{2}+\int_{0}^{T} \alpha(t)(1+G(u(t))) d t .
\end{aligned}
$$

In view of $\left(\mathrm{F}_{0}\right)$, conclusion (i) follows. On the other hand, conclusion (ii) follows from (i) and Theorem (2.1).

We close our discussion here by showing that the methods presented in Theorem (3.2) allow us to treat certain second order equations. Consider for example the following second order functional-differential equation

$$
\ddot{u}(t)=C g(u(t))+\int_{0}^{t} B(t-\tau) g(u(\tau)) d \tau+f(t),
$$

where $C$ is a bounded linear operator on $\mathscr{H}$ and $f$ is a mapping from $[0, \infty)$ into $\mathscr{H}$. Define a new transformation $A(t)$ by the expression

$$
A(t)=-\int_{0}^{t} B(\tau) d \tau-C .
$$

Using (3.9) we can rewrite (3.8) in the following form:

$$
\ddot{u}(t)=-A(0) g(u(t))-\int_{0}^{t} \dot{A}(t-\tau) g(u(\tau)) d \tau+f(t) .
$$

An integration of (3.10) will now yield

$$
\dot{u}(t)=\dot{u}(0)-\int_{0}^{t} A(t-\tau) g(u(\tau)) d \tau+\int_{0}^{t} f(\tau) d \tau,
$$

which is of the form (3.1) under investigation. In order to apply Theorem (3.2), we need to assume that $\int_{0}^{t} f(\tau) d \tau \in L_{1}[0, \infty),\left(\mathrm{G}_{2}\right)$ holds and $A(\infty)$ exists and is positive as given in Theorem (3.2). This will be the case if $B(t) \geqq 0$ (as a semidefinite operator) and $C-\int_{0}^{\infty} B(\tau) d \tau$ defines a positive definite operator. This investigation is carried out in $\$ 5$ and 6 where we also consider cases in which $A(\infty)=0$.

4. Positive and strongly positive kernels. In this section we give some sufficient conditions for positivity and strong positivity of kernels. Halanay [4] stated, but incorrectly proved, that in $\boldsymbol{R}^{1}, a(t)$ is strongly positive if

$$
(-1)^{k} a^{(k)}(t) \geqq 0, \quad k=0,1,2,
$$

and $a(t) \not \equiv$ constant. That (4.1) alone is sufficient for the positivity of $a(t)$ is well known (see Loève [11, p. 217]). Without additional assumptions on $a(t)$, one 
clearly cannot expect strong positivity. It is, however, interesting to find that under the additional assumption that $a(t) \not \equiv$ constant, $a(t)$ in fact defines a strongly positive kernel. The effort to verify Halanay's claim and clarify his proof led us to a result which is valid in general Hilbert space. In the special one dimensional case, we can prove a better result, motivated by the work of Hannsgen [5]. Later we show by examples that the monotonicity conditions are too special in that positivity and strong positivity may hold for some oscillatory kernels.

It is well known in circuit theory (see for example [13]) that a kernel $a(t)$ in $\boldsymbol{R}^{1}$ will define a positive kernel if its Laplace transform $\hat{a}(s)$ satisfies the condition

$$
\operatorname{Re} \hat{a}(s)>0 \quad \text { in } \operatorname{Re} s>0 \text {. }
$$

If $\hat{a}(s)$ exists for $\operatorname{Re} s=0$ and is continuous in $\operatorname{Re} s \geqq 0$ then it follows from (4.2) that $\operatorname{Re} \hat{a}(s) \geqq 0$ on $\operatorname{Re} s=0$. Our main result is that, apart from some technical details, strong positivity will hold if $\operatorname{Re} \hat{a}(s)$ is strictly positive on $\operatorname{Re} s=0$.

The general results concern a family $\tilde{A}=\{A(t): t \geqq 0\}$ of bounded linear transformations on a Hilbert space $\mathscr{H}$. For such a family we define the Laplace transform $\hat{A}(s)$ by the formula

$$
\hat{A}(s)=\lim _{T \rightarrow \infty} \int_{0}^{T} e^{-s t} A(t) d t
$$

Here we mean the Bochner integral and the uniform limit; all subsequent limits will be in the uniform topology.

We give here an outline of the ideas of the section. It appears that natural analogs of one dimensional results hold only when $A(t)$ is symmetric for each $t$. Under this assumption, and some more technical ones, we show that if $\operatorname{Re} \hat{A}(i \tau)$ is nonnegative semidefinite on $\mathscr{H}$ for each $\tau \in \boldsymbol{R}$ then $\tilde{A}$ is a positive kernel. This is an analog of (4.2). Then strong positivity will follow if we can find $S$ and $\varepsilon$, which are positive, such that if $\tilde{S}=\varepsilon e^{-S t}$, then

$$
\operatorname{Re}(\hat{A}(i \tau)-\hat{S}(i \tau)) \text { is positive for all } \tau \in R .
$$

We will, in fact, look for $S$ in the form $c I, c \geqq 0$. Then $\hat{S}=(s+c)^{-1} I$ and (4.4) becomes

$$
(\xi, \operatorname{Re} \hat{A}(i \tau) \xi) \geqq \varepsilon c\|\xi\|^{2} /\left(c^{2}+\tau^{2}\right) \quad \text { for all } \xi \in \mathscr{H} \text { and } \tau \in \boldsymbol{R} .
$$

The rather complicated work to follow aims at establishing the inequality (4.5). There are two difficulties. First we have to verify (4.5) for large $\tau$ and hence we must obtain estimates for $\hat{A}(i \tau)$ as $\tau \rightarrow \infty$. Second we must obtain the inequality for $\tau$ 's on bounded subsets. Here a distinction must be made between finite and infinite dimensional spaces. If $\operatorname{dim} \mathscr{H}<\infty$ and $\hat{A}(i \tau)$ is continuous and positive then (4.5) necessarily holds on bounded $\tau$ sets. In infinite dimensional spaces we must essentially postulate that (4.5) holds for $\tau$ in bounded subsets of $\boldsymbol{R}$. 
We impose on $A$ the conditions:

$\left(\mathrm{A}_{1}\right) A(t)$ is twice strongly differentiable on $[0, \infty)$, $\left(\mathrm{A}_{2}\right) \int_{0}^{\infty}\left\|A^{(k)}(t)\right\| d t<\infty, k=1,2$.

We have then

$$
\left\|A\left(t_{1}\right)-A\left(t_{2}\right)\right\|=\left\|\int_{t_{1}}^{t_{2}} A^{\prime}(t) d t\right\| \leqq \int_{t_{1}}^{t_{2}}\left\|A^{\prime}(t)\right\| d t
$$

and hence

$$
\lim _{t \rightarrow \infty} A(t)=A(\infty) \text { exists. }
$$

We set $A_{0}(t)=A(t)-A(\infty)$ and require that $A_{0}$ satisfy

$\left(\mathrm{A}_{3}\right) \int_{0}^{\infty}\left\|A_{0}(t)\right\| d t<\infty$.

For the Laplace transform $\hat{A}(s)$ of $A(t) \in \tilde{A}$, we have $\hat{A}(s)=s^{-1} \hat{A}(\infty)+\hat{A}_{0}(s)$ where $\hat{A}_{0}$ is the Laplace transform of $A_{0}$ and we collect here some facts concerning the transforms.

LeMmA (4.1). (1) $\hat{A}_{0}(\sigma+i \tau)$ exists and is continuous in $\sigma \geqq 0$. It is also holomorphic in $\sigma>0$.

(2) $\hat{A}(s)=s^{-1} A(0)+s^{-2} A^{\prime}(0)+o\left(s^{-2}\right)$ uniformly as $|s| \rightarrow \infty$ in $\sigma \geqq 0$.

Proof. (1) follows easily from $\left(\mathrm{A}_{3}\right)$. To prove (2) we integrate by parts twice, using $\left(\mathrm{A}_{1}\right)$, and obtain (note that $\left(\mathrm{A}_{2}\right)$ implies $A^{\prime}(\infty)$ exists and must be zero)

$$
\hat{A}(s)=s^{-1} A(0)+s^{-2} A^{\prime}(0)+s^{-2} \int_{0}^{\infty} e^{-s t} A^{\prime \prime}(t) d t .
$$

Conclusion (2) follows from an application of the Riemann-Lebesgue Lemma.

LEMMA (4.2). Let $\hat{A}_{0}(\sigma+i \tau)=\Phi(\sigma, \tau)+i \Psi(\sigma, \tau)$. Then

$$
A(t)-A(\infty)=\frac{2}{\pi} \int_{0}^{\infty} \Phi(0, \tau) \cos \tau t d \tau
$$

Proof. From Lemma (4.1) we observe that $\hat{A}_{0}$ satisfies the conditions

$$
\sup _{\sigma>0}\left\{\int_{-\infty}^{+\infty}\left\|\hat{A}_{0}(\sigma+i \tau)\right\|^{p} d \tau\right\}^{1 / p}<\infty
$$

$$
\lim _{\sigma \downarrow 0} \hat{A_{0}}(\sigma+i \tau)=\hat{A_{0}}(i \tau) \text { exists for all } \tau \text { and }\left\|\hat{A}_{0}(i \tau)\right\| \in L_{p}(-\infty, \infty) \text {, }
$$

for any $p>1$. It follows (see $\left[7\right.$, p. 227]) that $\hat{A_{0}}$ can be represented as a Cauchy integral of the form

$$
\hat{A_{0}}(\sigma+i \tau)=\frac{1}{\pi} \int_{-\infty}^{+\infty} \frac{\Phi(0, \eta) d \eta}{\sigma+i \tau-i \eta}
$$


Thus we can express $\Psi(0, \tau)$ in terms of $\Phi(0, \xi)$ by the formula

$$
\Psi(0, \tau)=-\frac{1}{\pi} \int_{-\infty}^{+\infty} \frac{\Phi(0, \eta)}{\tau-\eta} d \eta
$$

where the Cauchy principal value is meant in (4.10).

Now $A_{0}(t)$ may be recovered from $\hat{A}_{0}$ by the inversion formula (again see [7]),

$$
A_{0}(t)=\frac{1}{2 \pi} \int_{-\infty}^{+\infty} e^{i t \tau}(\Phi(0, \tau)+i \Psi(0, \tau)) d \tau
$$

Since $\hat{A}_{0}(s)$ is real for $\tau=0$ we must have $\Phi(0, \tau)=\Phi(0,-\tau)$ and $\Psi(0, \tau)$ $=-\Psi(0,-\tau)$ and hence $(4.11)$ can be written as

$$
A_{0}(t)=\frac{1}{2 \pi} \int_{-\infty}^{\infty}(\Phi(0, \tau) \cos \tau t-\Psi(0, \tau) \sin \tau t) d \tau
$$

We use (4.10) to rewrite the second integral as follows:

$$
\begin{aligned}
\int_{-\infty}^{+\infty} \Psi(0, \tau) \sin \tau t d \tau & =-\frac{1}{\pi} \int_{-\infty}^{+\infty} d \tau \sin \tau t \int_{-\infty}^{+\infty} \frac{\Phi(0, \eta)}{\tau-\eta} d \eta \\
& =-\frac{1}{\pi} \int_{-\infty}^{+\infty} \Phi(0, \eta) d \eta \int_{-\infty}^{+\infty} \frac{\sin \tau t}{\tau-\eta} d \tau
\end{aligned}
$$

The $\tau$ integral can be evaluated by contour integration to yield

$$
\int_{-\infty}^{+\infty} \Psi(0, \tau) \sin \tau t d \tau=-\int_{-\infty}^{\infty} \Phi(0, \eta) \cos \eta t d \eta
$$

If we substitute (4.13) into (4.12) and use the fact that $\Phi$ is even we obtain the formula in Lemma (4.2).

From Lemma (4.2) we obtain an analog of a part of Bochner's theorem in one dimension as expressed by condition (4.2).

LEMmA (4.3). Let $A(t)$ be symmetric for all $t \geqq 0$. Suppose further that $A(\infty)$ is positive semidefinite and $\operatorname{Re} \hat{A}_{0}(i \tau)$ is positive semidefinite for all $\tau$. Then $\tilde{A}$ defines a positive kernel.

Proof. Let us define $\bar{A}(t)$ for all $t$ by $\bar{A}(t)=A(t), t>0, \bar{A}(t)=A(-t)$ for $t$ negative. Consider then

$$
\begin{aligned}
\bar{Q}_{\tilde{A}}(\mu ; T) & =\int_{0}^{T}\left(\mu(t), \int_{0}^{t} \bar{A}(t-\tau) \mu(\tau) d \tau\right) d t \\
& =Q_{\tilde{A}}(\mu ; T)+\int_{0}^{T}\left(\mu(t), \int_{t}^{T} A(\tau-t) \mu(\tau) d \tau\right) d t \\
& =Q_{\tilde{A}}(\mu ; T)+\int_{0}^{T} \int_{0}^{\tau}(\mu(t), A(\tau-t) \mu(\tau)) d t d \tau
\end{aligned}
$$


Since $A(\tau-t)$ is symmetric, we may rewrite this as

$$
\bar{Q}_{\tilde{A}}(\mu ; T)=Q_{\tilde{A}}(\mu ; T)+\int_{0}^{T} \int_{0}^{t}(A(\tau-t) \mu(t), \mu(\tau)) d t d \tau=2 Q_{\tilde{A}}(\mu ; T) .
$$

We establish that $\bar{Q}_{\tilde{A}}(\mu ; T)$ must be positive. Note that

$$
\bar{Q}_{\tilde{A}}(\mu ; T)=\int_{0}^{T}\left(\mu(t), A(\infty) \int_{0}^{T} \mu(\tau) d \tau\right) d t+\int_{0}^{T}\left(\mu(t), \int_{0}^{T} A_{0}(t-\tau) \mu(\tau) d \tau\right) d t .
$$

From Lemma (4.2), we obtain then

$$
\begin{aligned}
& \bar{Q}_{\tilde{A}}(\mu ; T) \\
& \quad \geqq \frac{2}{\pi} \int_{0}^{T}\left(\mu(t), \int_{0}^{T} \int_{0}^{+\infty} \Phi(0, \eta) \mu(\tau)(\cos \eta t \cos \eta \tau+\sin \eta t \sin \eta \tau) d \eta d \tau\right) d t .
\end{aligned}
$$

We observe that

$$
\begin{aligned}
\int_{0}^{T}\left(\mu(t), \int_{0}^{T}\left(\int_{0}^{\infty} \Phi(0, \eta) \cos \eta t\right.\right. & \cos \eta \tau d \eta) \mu(\tau) d \tau) d t \\
& =\int_{0}^{T} \int_{0}^{T} \int_{0}^{\infty}(\mu(t) \cos \eta t, \Phi(0, \eta) \cos \eta \tau \mu(\tau)) d \eta d \tau d t \\
& =\int_{0}^{\infty}\left(\int_{0}^{T}\left(\mu(t) \cos \eta t, \int_{0}^{T} \Phi(0, \eta) \mu(\tau) \cos \eta \tau d \tau\right) d t\right) d \eta \\
& =\int_{0}^{\infty}\left(\int_{0}^{T} \mu(t) \cos \eta t d t, \Phi(0, \eta) \int_{0}^{T} \mu(\tau) \cos \eta \tau d \tau\right) d \eta
\end{aligned}
$$

This term is greater than or equal to zero since $\Phi(0, \eta)$ is positive. The sine terms are handled similarly and this completes the proof.

From now on we assume that the $A(t)$ are all symmetric. We give now a condition which will guarantee the positivity of $\hat{A}_{0}(i \tau)$.

THEOREM (4.1). Suppose that $\tilde{A}$ satisfies the conditions $\left({ }^{3}\right)$

$\left(\mathrm{A}_{4}\right)(-1)^{k} A^{(k)}(t)$ positive definite, $k=0,1$, for $t \geqq 0$,

( $\left.\mathrm{A}_{5}\right) A^{\prime}\left(t_{2}\right)-A^{\prime}\left(t_{1}\right)$ positive definite for $t_{2}>t_{1}$.

Then $\operatorname{Re} \hat{A}_{0}(i \tau)$ is positive definite for all $\tau \in \boldsymbol{R}$.

Proof. Observe that from $\left(\mathrm{A}_{4}\right)$

$$
\left(\xi, \hat{A}_{0}(0) \xi\right)=\int_{0}^{\infty}(\xi, A(t) \xi) d t \geqq 0,
$$

for all $\xi \in \mathscr{H}$. Suppose that for some $\xi$ the integral is zero. Hence $(\xi, A(t) \xi) \equiv 0$ and consequently $\xi=0$. On the other hand, for $\tau>0$, we have

( $\left.{ }^{3}\right)$ It will be clear from the proof that these conditions are slightly stronger than really needed. In particular in $R^{1}$ the positive definiteness in $\left(A_{4}\right)$ and $\left(A_{5}\right)$ can be relaxed to semidefiniteness together with $A(t) \not \equiv$ constant. 


$$
\begin{aligned}
\left(\xi, \operatorname{Re} \hat{A}_{0}(i \tau) \xi\right) & =\int_{0}^{\infty}\left(\xi, A_{0}(t) \xi\right) \cos \tau t d t \\
& =-\frac{1}{\tau} \int_{0}^{\infty}\left(\xi, A_{0}^{\prime}(t) \xi\right) \sin \tau t d t \\
& =-\frac{1}{\tau}\left\{\sum_{k=0}^{\infty} \int_{0}^{\pi / \tau} \sin \tau t\left(\xi,\left[A_{0}^{\prime}\left(t+\frac{2 k \pi}{\tau}\right)-A_{0}^{\prime}\left(t+\frac{(2 k+1) \pi}{\tau}\right)\right] \xi\right) d t\right\} .
\end{aligned}
$$

By $\left(\mathrm{A}_{5}\right)$ each term in the sum is positive unless $\xi=0$. The case $\tau<0$ follows by reflection since $\operatorname{Re} \hat{A}_{0}(i \tau)$ is an even function of $\tau$.

Theorem (4.1) and Lemma (4.3) yields immediately

COROllary (4.1). If $A(\infty)$ is positive semidefinite and $\tilde{A}$ satisfies $\left(\mathrm{A}_{4}\right)$ and $\left(\mathrm{A}_{5}\right)$ then $A$ is a positive kernel.

We are ready now to discuss strong positivity. As indicated earlier we try to find a semigroup of the form $e^{-c I t}, c>0$, such that

$$
\tilde{A}-e^{c I t} \text { is positive, }
$$

and, as we showed before, this will be true if we can establish the estimate (4.5). We start with a lemma.

Lemma (4.4). Suppose $A^{\prime}(0)$ is positive definite. Then there exists $M$ such that (4.5) is valid for all $\tau$ with $|\tau| \geqq M$.

Proof. Since (4.5) refers only to $\hat{A}_{0}$ it is no loss in generality to assume $A(\infty)=0$. Then we obtain from Lemma (4.1)

$$
(\xi, \Phi(0, \tau) \xi)=-\tau^{-2}\left[\left(\xi, A^{\prime}(0) \xi\right)+(\xi, R(\tau) \xi)\right],
$$

where $\|R(\tau)\|=o(1)$ as $|\tau| \rightarrow \infty$. It follows from $\left(\mathrm{A}_{4}\right)$ that, for some $m>0$, $(\xi, \Phi(0, \tau) \xi) \geqq\left(m / 2 \tau^{2}\right)\|\xi\|^{2}$ for $|\tau| \geqq M$. Thus (4.5) will be satisfied if we choose $\varepsilon=1$ and $c$ such that $c<m / 2$ where $M \geqq 1$.

From Lemma (4.4) and the remarks above we obtain the following conditional result.

THEOREM (4.2). Suppose $\tilde{A}$ satisfies $\left(\mathrm{A}_{1}\right)-\left(\mathrm{A}_{4}\right)$ and $A(t)$ is symmetric for each $t$. Then $\tilde{A}$ is strongly positive if it satisfies the following condition:

(4.15) Given any $M$ there exists an $\alpha>0$ such that $\left(\xi, \operatorname{Re} A_{0}(i \tau) \xi\right) \geqq \alpha\|\xi\|^{2}$ for $|\tau| \leqq M$.

Proof. Fix $M$ and $c$ as in Lemma (4.1) and choose $\varepsilon=\alpha c$.

Again we have an immediate corollary.

Corollary (4.2). If $\mathscr{H}$ is finite dimensional, the assumption (4.15) can be dropped. 
Proof. By Theorem (4.1), $\left(\xi, \operatorname{Re} A_{0}(i \tau) \xi\right)$ is positive definite for all $\tau$ in $|\tau| \leqq M$. Hence $\left(\xi, \operatorname{Re} A_{0}(i \eta) \xi\right)$ is a positive, continuous function on the compact set $(-M, M) \times U_{\mathscr{H}}$ where $U_{\mathscr{H}}$ is the unit sphere in $\mathscr{H}$. It follows that (4.15) is satisfied automatically.

We want to discuss in some detail the case of $\boldsymbol{R}^{1}$ where some of the conditions can be weakened a little. Let us suppose that $a$ is such that its transform has the form $a(\infty) / s+\hat{a}(s)$ where $\hat{a}(s)$ exists and is continuous in $\operatorname{Re} s \geqq 0$. Then, subject to certain technical details, the following statements will be true:

(1) If $a(\infty) \geqq 0$ and $\operatorname{Re} \hat{a}(i \tau) \geqq 0$ then $a$ is a positive kernel.

(2) If $\operatorname{Re} \hat{a}(i \tau) \geqq \varepsilon c /\left(c^{2}+\tau^{2}\right)$ for some $\varepsilon$ and $c$, positive, then $a$ is strongly positive.

(3) If $a(\infty) \geqq 0, \operatorname{Re} \hat{a}(i \tau)>0$ and $\operatorname{Re} \hat{a}(i \tau) \geqq b / \tau^{2}$, for $\mid \tau_{\mid}$sufficiently large, with $b$ some positive constant, then (2) is satisfied, hence $a$ is strongly positive.

These statements enable one to verify directly that certain simple functions are positive or strongly positive. For example one can show in this way that $t^{-\alpha} \cos w t$, $0 \leqq \alpha<1$, is positive while $t^{-\alpha} e^{-b t} \cos w t, 0 \leqq \alpha<1, b>0$, is strongly positive. It is interesting that $e^{-b t} \sin w t$ is not even positive, let alone strongly positive. (See [3].) These examples show that Halanay's result (condition (4.1)) is too special. We can have $a$ 's which are strongly positive and are both oscillatory and singular at zero. We now state and prove our version of Halanay's result.

THEOREM (4.3). Let a(t) satisfy the following conditions:

$\left(\mathrm{a}_{1}\right) a(t) \in C^{1}(0, \infty) \cap L_{1}(0,1)$,

$\left(\mathrm{a}_{2}\right) a(t) \geqq 0, a^{\prime}(t) \leqq 0$,

$\left(\mathrm{a}_{3}\right) a^{\prime}(t)$ nondecreasing,

$\left(\mathrm{a}_{4}\right) a(t) \not \equiv$ constant .

Then a(t) defines a strongly positive kernel.

Proof. We note that $\left(\mathrm{a}_{2}\right)$ implies that $a(\infty)$ exists and is greater than or equal to zero, and that $a(t)-a(\infty)$ again satisfies the assumptions $\left(\mathrm{a}_{1}\right)-\left(\mathrm{a}_{4}\right)$. It follows that the transform

$$
\hat{a}_{0}(s)=\lim _{T \rightarrow \infty} \int_{0}^{T} e^{-s t}(a(t)-a(\infty)) d t
$$

exists for $\operatorname{Re} s \geqq 0, s \neq 0$. (For $s=i \tau$, the integral is conditionally convergent.) We want to establish that

$$
\operatorname{Re} \hat{a}_{0}(i \tau)>0 \text { for all } \tau \in \boldsymbol{R}
$$

If $a(0)$ is finite, then this follows as in the proof of Theorem (4.1). If $a(0)=\infty$, the arguments need certain modifications. We write for $\tau>0$

$$
\operatorname{Re} \hat{a}_{0}(i \tau)=\left(\int_{0}^{2 \pi / \tau}+\int_{2 \pi / \tau}^{\infty}\right)(a(t)-a(\infty)) \cos \tau t d t
$$


The second integral above is nonnegative by the argument used in Theorem (4.1). For the first integral, we consider, for $0<\varepsilon<2 \pi / \tau$,

$$
\begin{aligned}
\int_{\varepsilon}^{2 \pi / \tau}(a(t)-a(\infty)) \cos \tau t d t= & -\frac{1}{\tau}(a(\varepsilon)-a(\infty)) \sin \varepsilon \tau-\frac{1}{\tau} \int_{\pi / \tau}^{\pi / \tau+\varepsilon} a^{\prime}(t) \sin \tau t d t \\
& -\frac{1}{\tau} \int_{\varepsilon}^{\pi / \tau}\left(a^{\prime}(t)-a^{\prime}(t+\pi / \tau)\right) \sin \tau t d t
\end{aligned}
$$

The last integral above is nonnegative by $\left(a_{3}\right)$. The first integral on the right tends to zero as $\varepsilon$ tends to zero. We claim that the first term on the right also tends to zero as $\varepsilon$ tends to zero. For $a(t)$ is nonincreasing and is locally in $L_{1}(0,1)$, hence we have $\lim _{\varepsilon \rightarrow 0} \varepsilon a(\varepsilon)=0$. Thus,

$$
\begin{aligned}
\operatorname{Re} \hat{a}_{0}(i \tau) & =\lim _{\varepsilon \rightarrow 0} \int_{\varepsilon}^{\infty}(a(t)-a(\infty)) \cos \tau t d t \\
& \geqq \lim _{\varepsilon \rightarrow 0}-\frac{1}{\tau} \int_{\varepsilon}^{\pi / \tau}\left(a^{\prime}(t)-a^{\prime}(t+\pi / \tau)\right) \sin \tau t d t
\end{aligned}
$$

Now since $a(0)=\infty$ and $\left(a_{3}\right),\left(a_{4}\right)$ hold, we see that the last integral above is positive, proving (4.17).

In order to establish strong positivity of $a(t)$ we need to show that

$$
\operatorname{Re} \hat{a}_{0}(i \tau) \geqq b / \tau^{2}, \quad b>0,
$$

for all $|\tau| \geqq N$, where $N$ is some large number. We show in fact under the present hypothesis that we have

$$
\operatorname{Re} \hat{a}(i \tau) \geqq c /|\tau|, \quad c>0,
$$

for large $\tau$. Denote $a_{0}(t)=a(t)-a(\infty)$ for short. We first note that in view of $\left(a_{2}\right),\left(a_{3}\right)$ and $\left(a_{4}\right)$

$$
\int_{0}^{\infty} a_{0}(t) \cos t d t=\tilde{a}_{0}>0
$$

Without loss of generality, we need only to consider $\tau \geqq N$. Observe that

$$
\operatorname{Re} \hat{a}_{0}(i \tau)=\int_{0}^{\infty} a_{0}(t) \cos \tau t d t=\frac{1}{\tau} \int_{0}^{\infty} a_{0}\left(\frac{\xi}{\tau}\right) \cos \xi d \xi
$$

We will have established (4.19) if we can show that the last integral in (4.21) is nondecreasing as $\tau$ increases. Integrating by parts, we have

$$
\begin{aligned}
\int_{0}^{\infty} a_{0}\left(\frac{\xi}{\tau}\right) \cos \xi d \xi & =-\frac{1}{\tau} \int_{0}^{\infty} a_{0}^{\prime}\left(\frac{\xi}{\tau}\right) \sin \xi d \xi \\
& =-\int_{0}^{\infty} a_{0}^{\prime}(t) \sin \tau t d t
\end{aligned}
$$


which is uniformly convergent (as an improper integral) for all $\tau \geqq 1$. Consider the differentiation under the integral sign

$$
\frac{d}{d \tau} \int_{0}^{\infty} a_{0}\left(\frac{\xi}{\tau}\right) \cos \xi d \xi=\int_{0}^{\infty}-a_{0}^{\prime}\left(\frac{\xi}{\tau}\right) \frac{\xi}{\tau^{2}} \cos \xi d \xi
$$

which will be justified if the integral on the right of (4.22) is uniformly convergent for all $\tau \geqq 1$. This is in fact true as the following arguments show. Consider

$$
\int_{1}^{T} d a^{\prime}(t)=T a^{\prime}(T)-a^{\prime}(1)-\int_{1}^{T} a^{\prime}(t) d t
$$

It follows from $\left(a_{3}\right)$ that the integral on the left has a negative limit (possibly $-\infty$ ) as $T$ tends to infinity. Hence, the same is true of $T a^{\prime}(T)$. But the limit of $T a^{\prime}(T)$ must be zero in order that $a^{\prime}$ be integrable on $[1, \infty)$. Thus the integral on the left also has a limit.

Next, we note that

$$
\begin{aligned}
\int_{0}^{\infty}-a_{0}^{\prime}\left(\frac{\xi}{\tau}\right) \frac{\xi}{\tau^{2}} \cos \xi d \xi & =\int_{0}^{\infty}-t a_{0}^{\prime}(t) \cos \tau t d t \\
& =\frac{1}{\tau}\left[\int_{0}^{\infty}\left(a^{\prime}(t) \sin \tau t\right) d t+\int_{0}^{\infty} t \sin \tau t d a^{\prime}(t)\right]
\end{aligned}
$$

Since $a^{\prime}$ is monotone and decreases to zero and the integral $\int_{1}^{\infty} t d a^{\prime}(t)$ exists, we conclude that the last integral in the above is uniformly convergent for $\tau \geqq 1$. By (4.22), (4.21) and (4.20), we obtain the desired estimate (4.19).

5. Equations in $\boldsymbol{R}^{1}$. We consider here nonlinear functional-differential equations in $\boldsymbol{R}^{1}$ of the form

$$
\dot{u}(t)=-\int_{0}^{t} a(t-\tau) g(u(\tau)) d \tau+f(t, u(t)),
$$

where $u(t), a(t)$ and $f(t, u(t))$ are continuous scalar functions. As special cases of (5.1), we have what we call the unperturbed equation in which $f(t, u(t))$ does not depend on $u$, that is,

$$
\dot{u}(t)=-\int_{0}^{t} a(t-\tau) g(u(\tau)) d \tau+f(t),
$$

and more specially the homogeneous equation

$$
\dot{u}(t)=-\int_{0}^{t} a(t-\tau) g(u(\tau)) d \tau .
$$

It was the study of these equations which first led us to the work of this paper. We mention four fundamental papers in this area. Levin [8] and Halanay [4] for equation (5.3), Levin and Nohel [10] for equations (5.1) and (5.2), and most recently Hannsgen [6] for equation (5.2). Our work extends and clarifies the ideas 
of Halanay. In contrast to the results given in the other three papers [6], [8], [10], we do not have to find Lyapunov functions.

We are interested in the boundedness and asymptotic stability of solutions of equation (5.1). A general principle, stated by Halanay, is that if $a(t)$ defines a positive kernel then we have boundedness. On the other hand, if $a(t)$ defines a strongly positive kernel, then we have asymptotic stability. Halanay's remarks were directed to the homogeneous equation (5.3). We shall show here that to a large extent his idea remains valid for the more general equation (5.1). The other three references assumed monotonicity conditions on $a(t)$ similar to condition (4.1), and thus, by Theorem (4.2), automatically insured strong positivity. It should be noted here that Levin [8] and Levin and Nohel [10] made the stronger assumption

$$
(-1)^{k} a^{(k)}(t) \geqq 0, \quad k=0,1,2,3, t \in C[0, \infty),
$$

and $a(t) \not \equiv$ constant. Hannsgen [6] assumed a condition slightly weaker than (4.1) in that $a(t)$ is nonnegative and convex downward. In fact, his condition is also weaker than the assumptions $\left(a_{1}\right),\left(a_{2}\right),\left(a_{3}\right)$ and $\left(a_{4}\right)$ in Theorem (4.3). We shall have occasion to compare Hannsgen's assumptions later in this section.

We emphasize that under the strong positivity assumption on $a(t)$, and assumptions $\left(\mathrm{G}_{0}\right)$ and $\left(\mathrm{F}_{0}\right)$ on $g$ and $f$, Theorem (3.1) provides a "conditional" result; namely, if $g(u(t))$ is bounded and uniformly continuous then $g(u(t))$ tends to zero. Thus all the additional hypotheses on $g$ and $f$ made in [6], [8] and [10] cited above, and which we also make, are just designed to achieve these boundedness and uniformity requirements. In view of Theorem (4.3), most of our results here are valid for kernels with a singularity at zero. Moreover, examples at the end of $\S 4$ suggest that the strong positivity assumption can hold for functions which oscillate in every neighborhood of $t=\infty$.

We begin by presenting a heuristic discussion of the linear version of (5.2), namely,

$$
\dot{u}(t)=-\int_{0}^{t} a(t-\tau) u(\tau) d \tau+f(t) .
$$

This discussion will serve to indicate what theorems ought to be true. In the linear case, we can apply the Laplace transform method. This gives the transform of the solution, $\hat{u}(s)$, in terms of the transforms of $a(t)$ and $f(t)$. Specifically, we have

$$
\hat{u}(s)=(u(0)+\hat{f}(s)) /(s+\hat{a}(s)) .
$$

We will need the following facts about the Laplace transform. Let $\varphi(t)$ be a function of $t$ and $\hat{\varphi}(s)$ its transform. Consider the following cases:

(i) $\varphi(t) \in L_{1}(0, \infty)$,

(ii) $\varphi(t)-\varphi(\infty) \in L_{1}(0, \infty)$. 
In the first case (i), $\hat{\varphi}(s)$ is analytic in $\operatorname{Re} s>0$ and continuous in $\operatorname{Re} s \geqq 0$. In case (ii), $\hat{\varphi}(s)$ is analytic in $\operatorname{Re} s>0$ and $\hat{\varphi}(s)-\varphi(\infty) / s$ is continuous in $\operatorname{Re} s \geqq 0$. There exist some theorems in the other direction. We do not go into the technical details here but the following principles are roughly true. If the transform $\hat{\varphi}(s)$ is analytic in $\operatorname{Re} s>0$ and continuous in $\operatorname{Re} s \geqq 0$, then $\varphi(t)$ tends to zero as $t$ tends to infinity. Similarly, if $\hat{\varphi}(s)$ is analytic in $\operatorname{Re} s>0$ and $\hat{\varphi}(s)-\varphi(\infty) / s$ is continuous in $\operatorname{Re} s \geqq 0$ then $\varphi(t)-\varphi(\infty) \rightarrow 0$ as $t \rightarrow \infty$. We use these two simple principles, together with (5.6), to deduce information about $u(t)$ for large $t$ from the behavior of $\hat{u}(s)$ near $s=0$. Since $s^{-1} \hat{u}(s)$ is the transform of $\int_{0}^{t} u(\tau) d \tau$, we can also deduce information about the integral. We discuss four cases separately.

Case (1). $a \in L_{1}, f \in L_{1}$. In this case $\hat{a}$ and $\hat{f}$ are regular at $s=0$. If $\hat{a}(0)=\int_{0}^{\infty} a(t) d t$ $\neq 0$, then $\hat{u}(s)$ is regular at 0 . Thus, $u(t) \rightarrow 0$ as $t \rightarrow \infty$. On the other hand

$$
s^{-1} \hat{u}(s)-\frac{u(0)+\hat{f}(0)}{s \hat{a}(0)}=\frac{s(u(0)+\hat{f}(0))+u(0)(\hat{a}(0)-\hat{a}(s))+\hat{a}(0)(\hat{f}(s)-\hat{f}(0))}{s \hat{a}(0)(s+\hat{a}(0))}
$$

which is regular at $s=0$, so that,

$$
\int_{0}^{t} u(\tau) d \tau \rightarrow \frac{u(0)+\hat{f}(0)}{\hat{a}(0)} \text { as } t \rightarrow \infty .
$$

Case (2). $a(\infty)>0, a-a(\infty) \in L_{1}, f \in L_{1}$. Here $\hat{f}$ is regular but $\hat{a}(s) \sim a(\infty) / s$ near $s=0$. It follows from (5.6) that $\hat{u}(s)$ is again regular at $s=0$. Moreover

$$
s^{-1} \hat{u}(s)=\frac{u(0)+\hat{f}(s)}{s\left(a(\infty) / s+\hat{a}_{0}(s)+s\right)}=\frac{u(0)+\hat{f}(s)}{a(\infty)+s \hat{a}_{0}(s)+s^{2}},
$$

where $\hat{a}_{0}(s)=\hat{a}(s)-a(\infty) / s$, which is regular near $s=0$. Since the function defined in (5.9) is also regular near $s=0$, we conclude that $u(t)$ and $\int_{0}^{t} u(\tau) d \tau$ tend to zero as $t$ tends to infinity.

Case (3). $a(\infty)>0, a-a(\infty) \in L_{1}, f-f(\infty) \in L_{1}$. Here both $\hat{a}$ and $\hat{f}$ have a simple pole at $s=0$. In this case, formula (5.6) shows that $\hat{u}$ is regular at $s=0$ so that $u(t) \rightarrow 0$ as $t \rightarrow \infty$. On the other hand, corresponding to (5.9) we have

$$
s^{-1} \hat{u}(s)=\frac{u(0)+f_{0}(s)+f(\infty) / s}{s\left(a(\infty) / s+\hat{a}_{0}(s)+s\right)} \sim \frac{f(\infty)}{s a(\infty)} \quad \text { as } s \rightarrow 0 .
$$

Thus we have

$$
\int_{0}^{t} u(\tau) d \tau \rightarrow \frac{f(\infty)}{a(\infty)}
$$

Case (4). $a \in L_{1}, f-f_{\infty} \in L_{1}$. Here $\hat{a}$ is regular at zero but $\hat{f} \sim f(\infty) / s$ near $s=0$. A simple check with (5.6) yields $\hat{u}(s) \sim f(\infty) s^{-1} \hat{a}(0)$ as $s \rightarrow 0$. Hence

$$
u(t) \rightarrow f(\infty) / \hat{a}(0) \quad \text { as } t \rightarrow \infty .
$$

These conclusions for equation (5.5) can all be made rigorous but we choose to forego these details since we aim at generalizations for the nonlinear equation (5.1). Note that the assumptions that $a \in L_{1}$ and $a-a_{\infty} \in L_{1}$ are merely used to guarantee 
the existence of the Laplace transform $\hat{a}(s)$ on $\{s: \operatorname{Re} s \geqq 0\}$. In fact, most of these conclusions remain valid under conditions weaker than $\left(a_{1}\right),\left(a_{2}\right),\left(a_{3}\right)$ and $\left(a_{4}\right)$ of Theorem (4.3).

We list here the various conclusions concerning boundedness $\left(B_{k}\right)$ and stability $\left(\mathrm{S}_{k}\right)$ of all solutions of $(5.1)$ :

$\left(\mathrm{B}_{0}\right)|G(u(t))|$ bounded for $t \geqq 0, G(u)=\int_{0}^{u} g(\xi) d \xi$,

(B) $|g(u(t))|$ bounded for $t \geqq 0$,

(B) $Q_{\tilde{A}}[g(u) ; T]$ bounded for $T \geqq 0$,

$\left(\mathrm{B}_{3}\right)^{k}\left|u^{(k)}(t)\right|$ bounded for $t \geqq 0, k=0,1,2$.

( $\left.\mathrm{S}_{0}\right) \quad g(u(t)) \rightarrow 0$ as $t \rightarrow \infty$,

$\left(\overline{\mathrm{S}}_{0}\right) \quad g(u(t)) \rightarrow \alpha_{0} \neq 0$ as $t \rightarrow \infty$,

$\left(\mathrm{S}_{1}\right)^{k} u^{(k)}(t) \rightarrow 0$ as $t \rightarrow \infty, k=0,1,2$,

$\left(\mathrm{S}_{2}\right) \quad \int_{0}^{t} g(u(\tau)) d \tau \rightarrow 0$ as $t \rightarrow \infty$,

$\left(\overline{\mathrm{S}_{2}}\right) \quad \int_{0}^{t} g(u(\tau)) d \tau \rightarrow \alpha_{1} \neq 0$ as $t \rightarrow \infty$.

We shall need a number of hypotheses on $a, g$ and $f$ in addition to the assumptions $\left(\mathrm{a}_{1}\right),\left(\mathrm{a}_{2}\right),\left(\mathrm{a}_{3}\right),\left(\mathrm{a}_{4}\right)$ (which guarantee strong positivity of $a$ ). The assumptions on $a$ are

(a) $a \in L_{1}[0, \infty)$,

$\left(\mathrm{a}_{6}\right) a(\infty)>0$,

$\left(\mathrm{a}_{7}\right) a(\infty)>0, a-a(\infty) \in L_{1}[0, \infty)$,

(a) $a^{\prime} \in L_{1}[0, \infty)$

$\left(\mathrm{a}_{9}\right) \int_{t}^{\infty} a(\tau) d \tau \in L_{1}[0, \infty)$.

The hypotheses we will need with regard to $g$ and $f$ are

$\left(\mathrm{G}_{0}\right) G(u)=\int_{0}^{u} g(\xi) d \xi$ satisfies $\inf G(u)>-\infty$,

$\left(\mathrm{G}_{1}\right) G(u) \rightarrow \infty$ as $|u| \rightarrow \infty$,

$\left(\mathrm{G}_{2}\right) \mathrm{g} \in C(-\infty, \infty)$,

$\left(\mathrm{G}_{3}\right) g \in C(-\infty, \infty), u g(u)>0, u \neq 0$,

$\left(\mathrm{G}_{4}\right)|g(u)| \leqq M(1+G(u))$ for all $u$,

$\left(\mathrm{G}_{5}\right) g^{\prime}(\xi)>0$ for all $\xi \in \boldsymbol{R}$.

$\left(\mathrm{F}_{0}\right)$ There exists a function $\alpha(t) \in L_{1}[0, \infty)$ such that for all $t \geqq 0$ and all $|v|<\infty$, $|f(t, v) g(v)| \leqq \alpha(t)(1+G(v))$,

$\left(\mathrm{F}_{1}\right) f(t) \in L_{1}(0, \infty)$

$\left(\mathrm{F}_{2}\right)|f(t, u)|,\left|f_{t}(t, u)\right|,\left|f_{u}(t, u)\right|$ bounded for all $t \geqq 0$ and for every compact interval with respect to $u$.

We will have occasion to use the hypothesis that a solution and its derivatives $u^{(k)}(t)$ are uniformly continuous. For convenience, we also introduce the following abbreviations:

$(\mathrm{U})^{k} u^{(k)}(t)$ is uniformly continuous on $[0, \infty), k=0,1,2$.

To simplify the statements in this section, we assume without further mention that $a(t)$ defines a strongly positive kernel. Thus, as a consequence of Theorem (4.3) any kernel $a(t)$ satisfying assumptions $\left(\mathrm{a}_{1}\right)-\left(\mathrm{a}_{4}\right)$ defines a strongly positive kernel. 
The following lemma will be used in the proofs of a number of stability results concerning (5.1). We state these technical assertions explicitly here for easy reference. We comment that although the lemma extends completely to finite dimensional spaces only (i) and (iii) will have extensions to infinite dimensional spaces.

Lemma (5.1). (i) $\left(\mathrm{B}_{0}\right)$ and $\left(\mathrm{G}_{1}\right) \Rightarrow\left(\mathrm{B}_{3}\right)^{0}$.

(ii) $\left(\mathrm{B}_{0}\right)$ and $\left(\mathrm{G}_{1}\right),\left(\mathrm{G}_{2}\right) \Rightarrow\left(\mathrm{B}_{1}\right)$.

(iii) $\left(\mathrm{B}_{3}\right)^{k+1} \Rightarrow(\mathrm{U})^{k}, k=0,1$.

(iv) $\left(\mathrm{B}_{3}\right)^{0}$ and $(\mathrm{U})^{1} \Rightarrow\left(\mathrm{B}_{3}\right)^{1}$.

(v) $\left(\mathrm{S}_{1}\right)^{0}$ and $(\mathrm{U})^{1} \Rightarrow\left(\mathrm{S}_{1}\right)^{1}$.

(vi) $\left(\mathrm{S}_{0}\right)$ and $\left(\mathrm{G}_{3}\right) \Rightarrow\left(\mathrm{S}_{1}\right)^{0}$.

Proof. Only (iv) and (v) need proof. Since their proofs are similar, we show only (iv). Suppose that the conclusion is false, then there exists a sequence $\left\{t_{n}\right\} \in[0, \infty)$ such that $\left|\dot{u}\left(t_{n}\right)\right| \geqq 2 n$. By $(\mathrm{U})^{1}$, there exists $\delta>0$ such that $\left|\dot{u}\left(t_{n}\right)\right|>n$ for $t \in\left[t_{n}-\delta, t_{n}\right]$. Then by the mean value theorem, $\left|u\left(t_{n}\right)-u\left(t_{n}-\delta\right)\right| \geqq n \delta$ contradicting the assumption $\left(\mathrm{B}_{3}\right)^{0}$.

We are now ready to apply the weak stability principle, Theorem (3.1), to obtain stability results for solutions of (5.1). The first result is that of boundedness which follows from Theorem (3.1) and Corollary (3.1).

THEOREM (5.1). Let $a(t)$ be a positive kernel, and assume that $\left(\mathrm{G}_{0}\right),\left(\mathrm{G}_{1}\right),\left(\mathrm{G}_{2}\right)$ hold. Then

(i) for equation (5.1), $\left(\mathrm{F}_{0}\right) \Rightarrow\left(\mathrm{B}_{0}\right),\left(\mathrm{B}_{1}\right),\left(\mathrm{B}_{2}\right)$ and $\left(\mathrm{B}_{3}\right)^{0}$;

(ii) for equation (5.2), $\left(\mathrm{F}_{1}\right)$ and $\left(\mathrm{G}_{4}\right) \Rightarrow\left(\mathrm{B}_{0}\right),\left(\mathrm{B}_{1}\right),\left(\mathrm{B}_{2}\right)$ and $\left(\mathrm{B}_{3}\right)^{0}$;

(iii) for equation (5.3), $\left(\mathrm{B}_{0}\right),\left(\mathrm{B}_{1}\right),\left(\mathrm{B}_{2}\right)$ and $\left(\mathrm{B}_{3}\right)^{0}$ hold and we have in addition

(a) $\left(a_{5}\right) \Rightarrow\left(B_{3}\right)^{1}$,

(b) $\left(a_{8}\right) \Rightarrow\left(B_{3}\right)^{2}$.

ReMarK (5.1). Conclusion (ii) is contained in Levin and Nohel [10] under the stronger assumption (5.4) and is extended by Hannsgen [6] to kernels $a(t)$ satisfying $\left(a_{1}\right)-\left(a_{4}\right)$, and also $\left(a_{8}\right)$. (Hannsgen's condition does not assume that $a^{\prime}(t)$ exists everywhere but requires that $a(t)$ is not a function piecewise linear in a certain way. This condition is weaker than $\left(a_{1}\right)-\left(a_{4}\right)$. Hannsgen shows that if $a(t)$ is piecewise linear in a certain way then the solution is asymptotic to either a sine or cosine function at infinity, hence asymptotic stability does not hold in this case.) It should perhaps be pointed out that results given in [6] and [10] concern equation (5.2) with an additional term $b(t)$. This term is roughly a constant when $a(\infty)>0$, otherwise, $b(t) \in L_{1}[0, \infty)$. We return to this slightly more general case at the end of the section.

Theorem (5.1) provides the necessary boundedness condition on the solution for the application of Theorem (3.1). We now prove a lemma which isolates the necessary assumption for the solution to be uniformly continuous. 
LEMma (5.2). Let a be monotone decreasing and satisfy $\left(\mathrm{a}_{1}\right)$. Suppose that $u(t)$ is a solution of $(5.1)$ satisfying $\left(\mathrm{B}_{1}\right)$ and $\left(\mathrm{B}_{3}\right)^{0}$ and furthermore

$\left(\mathrm{U}_{f}\right)$ the function $f(t, u(t))$ is uniformly continuous on $[T, \infty)$, for some $T \geqq 0$.

Then $u(t)$ satisfies $(\mathrm{U})^{0}$.

Proof. In view of Lemma (5.1)(iv), we need only prove (U) ${ }^{1}$. From (5.1), for $t_{2}>t_{1}$, we have

$$
\begin{aligned}
\dot{u}\left(t_{2}\right)-\dot{u}\left(t_{1}\right)= & -\int_{0}^{t_{1}}\left(a\left(t_{2}-\tau\right)-a\left(t_{1}-\tau\right)\right) g(u(\tau)) d \tau \\
& -\int_{t_{1}}^{t_{2}} a\left(t_{2}-\tau\right) g(u(\tau)) d \tau+f\left(t_{2}, u\left(t_{2}\right)\right)-f\left(t_{1}, u\left(t_{1}\right)\right) .
\end{aligned}
$$

Let $\bar{g}=\sup _{[T, \infty)}|g(u(t))|$, which is finite by $\left(B_{1}\right)$. The second term on the right of (5.13) is clearly uniformly small with $t_{2}-t_{1}$ small on $[T, \infty)$. The third term is uniformly continuous by hypothesis. The first term is dominated, in absolute value, by

$$
\bar{g} \int_{0}^{t_{1}}\left(a\left(t_{1}-\tau\right)-a\left(t_{2}-\tau\right)\right) d \tau \leqq \bar{g}\left[\int_{0}^{t_{2}-t_{1}} a(\mu) d \mu-\int_{t_{1}}^{t_{2}} a(\mu) d \mu\right]
$$

and each term is again uniformly small with $t_{2}-t_{1}$ in $t_{1} \geqq T$.

Theorem (5.1) and Lemma (5.2), together with the weak stability principle, Theorem (2.1), give asymptotic stability of equation (5.1) provided that, for each solution $u(t)$, condition $\left(\mathrm{U}_{f}\right)$ is satisfied. For equation (5.3), the homogeneous equation, condition $\left(\mathrm{U}_{f}\right)$ is trivially satisfied. For the unperturbed equation (5.2), condition $\left(\mathrm{U}_{f}\right)$ is satisfied if and only if the function $f(t)$ is uniformly continuous. For the general equation (5.1), we need the additional assumption $\left(F_{2}\right)$. We summarize this basic stability result as follows:

TheOREM (5.2). Suppose that $\left(\mathrm{a}_{1}\right)-\left(\mathrm{a}_{4}\right),\left(\mathrm{G}_{1}\right)$ and $\left(\mathrm{G}_{3}\right)$ hold. Then we have

(i) for equation (5.1), $\left(\mathrm{a}_{5}\right),\left(\mathrm{F}_{0}\right),\left(\mathrm{F}_{2}\right) \Rightarrow\left(\mathrm{S}_{0}\right),\left(\mathrm{S}_{1}\right)^{0}$;

(ii) for equation (5.2), $\left(\mathrm{F}_{1}\right),\left(\mathrm{G}_{4}\right) \Rightarrow\left(\mathrm{S}_{0}\right),\left(\mathrm{S}_{1}\right)^{0}$;

(iii) for equation (5.3), $\left(\mathrm{S}_{1}\right)^{0}$ holds.

Proof. Since (ii) and (iii) are simple consequences of (i), we need prove only (i). In view of Lemma (5.2), it suffices to show that condition $\left(U_{f}\right)$ is satisfied for every solution $u(t)$. In order to show this we first prove that $u(t)$ satisfies $\left(\mathrm{B}_{3}\right)^{1}$. Observe that Theorem (5.1) implies $\left(B_{1}\right)$. This, together with $\left(a_{5}\right)$ and $\left(F_{2}\right)$ (the boundedness of $f$ ) yields $|\dot{u}(t)| \leqq B$. Next, we see that, for any $t_{1}, t_{2} \geqq 0$,

$$
\begin{aligned}
\left|f\left(t_{2}, u\left(t_{2}\right)\right)-f\left(t_{1}, u\left(t_{1}\right)\right)\right| & \leqq\left|f\left(t_{2}, u\left(t_{2}\right)\right)-f\left(t_{1}, u\left(t_{2}\right)\right)\right|+\left|f\left(t_{1}, u\left(t_{2}\right)\right)-f\left(t_{1}, u\left(t_{1}\right)\right)\right| \\
& \leqq M\left|t_{2}-t_{1}\right|+M\left|u\left(t_{2}\right)-u\left(t_{1}\right)\right| .
\end{aligned}
$$

Since $\left(B_{3}\right)^{1} \Rightarrow(U)^{0}$, this proves $\left(U_{f}\right)$ and hence completes the proof.

We note that if $\left(G_{3}\right)$ is replaced by the weaker assumptions $\left(G_{0}\right)$ and $\left(G_{2}\right)$ in the above result, then the conclusion is that all solutions satisfy $\left(\mathrm{S}_{0}\right)$ instead of $\left(\mathrm{S}_{1}\right)^{0}$. As a simple example of a function $f(t, u)$ satisfying $\left(\mathrm{F}_{0}\right)$ and $\left(\mathrm{F}_{2}\right)$ with respect to $g$ 
satisfying $\left(\mathrm{G}_{1}\right)$ and $\left(\mathrm{G}_{3}\right)$, we take $f(t, u)=\alpha(t) H(u)$ where $\alpha(t) \in L_{1}[0, \infty), H(u)$ is sublinear in the sense that $|H(u)| \leqq K(1+|u|)$, and $g(u)=(\operatorname{sgn} u)|u|^{k}, k>0$.

REMARK (5.2). Levin [8] proved Theorem (5.2)(iii) for the homogeneous equation (5.3) under the stronger assumption (5.4). Hannsgen [6] improved Levin's result, under assumptions slightly weaker than $\left(a_{1}\right)-\left(a_{4}\right)$, in that no differentiability requirement on $a(t)$ is made. Hannsgen's proof is based on showing that $\dot{u}(t)$ is bounded. He assumed either (i) $a(0)<\infty$ and $|\dot{f}(t)|$ bounded or (ii) (a $\left.\mathrm{a}_{5}\right)$ and $|f(t)|$ bounded. The boundedness of $|\dot{u}(t)|$ would of course imply $(\mathrm{U})^{0}$. Our point here is that uniform continuity of $u(t)$ itself is enough hence we obtain the slightly stronger result as summarized in Theorem (5.2) above.

In the case of the unperturbed equation (5.2) the asymptotic stability of $g(u(t))$ together with some additional assumptions on $a$ and $f$ will yield the stability results $\left(\mathrm{S}_{1}\right)^{k}$ with $k=1,2$.

TheOREM (5.3). Let $\left(\mathrm{a}_{1}\right)-\left(\mathrm{a}_{4}\right),\left(\mathrm{G}_{1}\right),\left(\mathrm{G}_{3}\right),\left(\mathrm{G}_{4}\right)$ and $\left(\mathrm{F}_{1}\right)$ hold. If $f(t)$ is uniformly continuous, then every solution $u(t)$ satisfies $\left(\mathrm{S}_{1}\right)^{1}$. If in addition $\left(\mathrm{a}_{8}\right)$ holds and $\dot{f}(t) \rightarrow 0$ as $t \rightarrow \infty$, then $u(t)$ satisfies $\left(\mathrm{S}_{1}\right)^{2}$.

Proof. We note that from the proof of Lemma (5.2), every solution satisfies $(U)^{1}$. Thus, it follows from Lemma $(5.1)(v)$ that every solution satisfies $\left(S_{1}\right)^{1}$. Next, we differentiate (5.2) to obtain

$$
\ddot{u}(t)=-a(0) g(u(t))-\int_{0}^{t} a^{\prime}(t-\tau) g(u(\tau)) d \tau+\dot{f}(t) .
$$

(Note that $a(0)$ exists and is finite.) By Theorem (5.2), every solution satisfies $\left(\mathrm{S}_{0}\right)$. Thus the first term on the right tends to zero. The third term tends to zero by hypothesis. To estimate the second term, we proceed as follows:

$$
\begin{aligned}
\left|\int_{0}^{t} a^{\prime}(t-\tau) g(u(\tau)) d \tau\right| & \leqq\left|\left(\int_{0}^{T}+\int_{0}^{t}\right) a^{\prime}(t-\tau) g(u(\tau)) d \tau\right| \\
& \leqq \sup _{t \in[0, T]}|g(u(t))| \int_{0}^{T}\left|a^{\prime}(t-\tau)\right| d \tau+\sup _{t \in[T, \infty)}|g(u(t))|(a(t)-a(T)) \\
& =\sup _{t \in[0, T]}|g(u(t))| \int_{t-T}^{t}\left|a^{\prime}(\sigma)\right| d \sigma+\sup _{t \in[T, \infty)}|g(u(t))|(a(t)-a(T)) .
\end{aligned}
$$

Since $a^{\prime} \in L_{1}$ we can make the integral small for fixed $T$ by choosing $t$ large. On the other hand, by $\left(\mathrm{S}_{0}\right)$, the second term can be made small by choosing $T$ sufficiently large. Hence the desired conclusion follows.

RemarK (5.3). Conclusions concerning $\left(\mathrm{S}_{1}\right)^{k}, k=1,2$, were obtained by Levin [8] for the homogeneous equation (5.3) under the stronger assumption (5.4). Results concerning $\left(\mathrm{S}_{1}\right)^{k}, k=1,2$, and $\left(\mathrm{S}_{2}\right)$ for equation (5.2) seem to be given here for the first time.

Hypothesis $\left(\mathrm{F}_{0}\right),\left(\mathrm{F}_{1}\right)$ are analogous to the assumption that $f \in L_{1}$ for the linear equation (5.5). Thus, Theorem (5.2) may be considered as a generalization of cases 
one and two discussed earlier in this section. We now present some results in these cases corresponding to the conclusions about the integral of the solution. Here, as in the linear case, the distinction between $a(\infty)>0$ or $a(\infty)=0$ becomes important. For simplicity, we treat only (5.2).

THEOREM (5.4). Let $\left(\mathrm{a}_{1}\right)-\left(\mathrm{a}_{4}\right),\left(\mathrm{G}_{1}\right),\left(\mathrm{G}_{3}\right),\left(\mathrm{G}_{4}\right)$ and $\left(\mathrm{F}_{1}\right)$ hold and $f(t)$ be uniformly continuous. Then for any solution of (5.2), we have

(i) $\left(\mathrm{a}_{7}\right) \Rightarrow\left(\mathrm{S}_{2}\right)$,

(ii) $\left(\mathrm{a}_{5}\right) \Rightarrow\left(\overline{\mathrm{S}}_{2}\right)$, and in particular

$$
\lim _{t \rightarrow \infty} \int_{0}^{t} g(u(\tau)) d \tau=\left(u(0)+\int_{0}^{\infty} f(t) d t\right) / \int_{0}^{\infty} a(t) d t .
$$

Proof. (i) Since $f(t)$ is uniformly continuous and satisfies $\left(\mathrm{F}_{1}\right)$, it must be so that $f(t) \rightarrow 0$ as $t \rightarrow \infty$. By Theorem (5.3), $u(t)$ satisfies $\left(\mathrm{S}_{1}\right)^{1}$. Write (5.1) as

$$
a(\infty) \int_{0}^{t} g(u(\tau)) d \tau=-\dot{u}(t)+f(t)-\int_{0}^{t}(a(t-\tau)-a(\infty)) g(u(\tau)) d \tau .
$$

Theorem (5.2) shows that $g(u(t)) \rightarrow 0$. Then the argument just used in the proof of Theorem (5.3) shows that the last term in (5.16) tends to zero as $t$ tends to infinity. By hypothesis, $a(\infty)>0$, thus we obtain from (5.16) the desired conclusion that $\int_{0}^{t} g(u(\tau)) d \tau \rightarrow 0$ as $t \rightarrow \infty$.

(ii) The proof given here is essentially the same as that of Levin [8] in the linear case. We reproduce it here. Let $w(t)=\int_{0}^{t} g(u(\tau)) d \tau$. We integrate (5.2) to obtain

$$
\begin{aligned}
u(t)-u(0)-\int_{0}^{t} f(\tau) d \tau & =-\int_{0}^{t} a(\tau-\sigma) g(u(\sigma)) d \sigma \\
& =-\int_{0}^{t} g(u(\sigma)) \int_{0}^{t-\sigma} a(\tau) d \tau=-\int_{0}^{t} a(t-\tau) w(\tau) d \tau .
\end{aligned}
$$

We assert first that $w(t)$ must remain bounded. Suppose not; then there must exist a sequence $\left\{t_{n}\right\}$ such that $t_{n} \rightarrow \infty$ and $\left|w\left(t_{n}\right)\right| \uparrow \infty$ with $|w(t)| \leqq\left|w\left(t_{n}\right)\right|$ for $0 \leqq t \leqq t_{n}$. Since $g(u(t))=w^{\prime}(t)$ is bounded (by Theorem (5.1)), w(t) is uniformly continuous and hence there exists a sequence $T_{n}, T_{n} \rightarrow \infty, T_{n} \leqq t_{n}$, such that $|w(t)| \geqq \frac{1}{2}\left|w\left(t_{n}\right)\right|$ in $\left[t_{n}-T_{n}, t_{n}\right]$. In particular, $w(t)$ and $w\left(t_{n}\right)$ have the same sign on these intervals. From (5.17), it follows that

$$
u\left(t_{n}\right)-u(0)-\int_{0}^{t_{n}} f(t) d t=-\int_{0}^{t_{n}-T_{n}} a\left(t_{n}-\tau\right) w(\tau) d \tau-\int_{t_{n}-T_{n}}^{t_{n}} a\left(t_{n}-\tau\right) w(\tau) d \tau
$$

The two integrals on the right of (5.18) may be estimated as follows:

$$
\begin{aligned}
\left|\int_{0}^{t_{n}-T_{n}} a\left(t_{n}-\tau\right) w(\tau) d \tau\right| & \leqq\left|w\left(t_{n}\right)\right| \int_{T_{n}}^{\infty} a(\tau) d \tau, \\
\left|\int_{t_{n}-T_{n}}^{t_{n}} a(t-\tau) w(\tau) d \tau\right| & \geqq \frac{1}{2}\left|w\left(t_{n}\right)\right| \int_{0}^{T_{n}} a(\tau) d \tau .
\end{aligned}
$$


Thus, (5.18) gives

$$
\left|u\left(t_{n}\right)-u(0)-\int_{0}^{t_{n}} f(t) d t\right| \geqq \frac{1}{2}\left|w\left(t_{n}\right)\right|\left\{\int_{0}^{T_{n}} a(\tau) d \tau-2 \int_{T_{n}}^{\infty} a(\tau) d \tau\right\} .
$$

The left-hand side of (5.19) is bounded by $\left(F_{1}\right)$ and Theorem (5.1), but the righthand side tends to infinity, which is impossible. Hence $|w(t)|$ must be bounded. We now rewrite (5.18) as

$$
\begin{aligned}
u\left(t_{n}\right)-u(0)-\int_{0}^{t_{n}} f(t) & d t \\
= & -\int_{0}^{t_{n}-T_{n}} a\left(t_{n}-\tau\right) w(\tau) d \tau-\int_{t_{n}-T_{n}}^{t} a\left(t_{n}-\tau\right)\left(w(\tau)-w\left(t_{n}\right)\right) d \tau \\
& -w\left(t_{n}\right) \int_{t_{n}-T_{n}}^{t_{n}} a\left(t_{n}-\tau\right) d \tau .
\end{aligned}
$$

Since $w(t)$ is bounded, we see that $\left(\mathrm{a}_{5}\right)$ implies that the first two terms on the right of (5.20) tend to zero as $t \rightarrow \infty$. Theorem (5.2) is applicable here, hence $\lim _{n \rightarrow \infty} u\left(t_{n}\right)=0$ and

$$
\lim _{n \rightarrow \infty} w\left(t_{n}\right)=\left(u(0)+\int_{0}^{\infty} f(t) d t\right) / \int_{0}^{\infty} a(t) d t .
$$

Since $w(t)$ is uniformly continuous, this proves (5.15).

REMARK (5.4). Theorem (5.4)(ii) in the special case when $g$ is linear and $f(t) \equiv 0$ is proved by Levin [8] under the stronger assumption (5.4).

Theorems (5.2), (5.3) and (5.4) give fairly complete analogs of the results for Cases (1) and (2) of the linear equation (5.5). We proceed to give analogs of Cases (3) and (4) in which the perturbing term $f(t, u)$ contains a constant term. These come from Theorem (3.1), its corollary, and Theorem (3.2). We restrict ourselves to the discussion of the unperturbed equation (5.2) in the following form:

$$
\dot{u}(t)=-\int_{0}^{t} a(t-\tau) g(u(\tau)) d \tau+f(\infty)+f_{1}(t),
$$

where $f_{1}(t) \in L_{1}[0, \infty)$. Similar conclusions for the more general equation (5.1) can be obtained under suitable assumptions. The result corresponding to Case (3) of the linear equation (5.5) is

ThEOREM (5.5). Suppose that $\left(\mathrm{a}_{1}\right)-\left(\mathrm{a}_{4}\right),\left(\mathrm{a}_{6}\right),\left(\mathrm{a}_{8}\right),\left(\mathrm{G}_{1}\right),\left(\mathrm{G}_{3}\right),\left(\mathrm{G}_{4}\right)$ hold, and moreover that $\left|f_{1}(t)\right|$ is bounded. Then any solution of $(5.21)$ satisfies $\left(\mathrm{B}_{0}\right),\left(\mathrm{B}_{1}\right),\left(\mathrm{B}_{3}\right)^{k}$, $k=0,1,2,\left(\mathrm{~S}_{0}\right),\left(\mathrm{S}_{1}\right)^{k}, k=0,1$, and $\left(\overline{\mathrm{S}}_{2}\right)$. In particular, we have

$$
\int_{0}^{t} g(u(\tau)) d \tau \rightarrow \frac{f(\infty)}{a(\infty)}
$$

Proof. We observe that $f(t)=f(\infty)+f_{1}(t), f_{1} \in L_{1}$ together with $\left(\mathrm{G}_{4}\right)$ imply that $\left(\tilde{F}_{0}\right)$ of $\S 3$ holds, as does $\left(G_{0}\right)$. Theorem (3.2) then yields $\left(B_{0}\right),\left(B_{3}\right)^{0},\left(B_{1}\right)$ and $\left(B_{2}\right)$ for $a(t)-a(\infty)$. 
To get uniform continuity of $u(t)$, we differentiate (5.21) and obtain

$$
\ddot{u}(t)=a(0) g(u(t))-\int_{0}^{t} a^{\prime}(t-\tau) g(u(\tau)) d \tau+\dot{f_{1}}(t) .
$$

Note that $a(0)$ exists on account of $\left(\mathrm{a}_{8}\right)$. Therefore, it follows from $\left(\mathrm{B}_{2}\right),\left(\mathrm{a}_{8}\right)$ and the boundedness of $\dot{f}(t)$ that $|\ddot{u}(t)|$ is bounded which implies (U) ${ }^{1}$. Lemma (5.1)(iv) gives $(\mathrm{U})^{0}$ as well as $\left(\mathrm{B}_{3}\right)^{1}$. Now $\left(\mathrm{B}_{2}\right)$ and Theorem (3.1) give $\left(\mathrm{S}_{0}\right)$ which implies $\left(\mathrm{S}_{1}\right)^{0}$ by $\left(\mathrm{G}_{3}\right)$. Since $(U)^{1}$ holds, we also have $\left(\mathrm{S}_{1}\right)^{1}$. At this point, we wish to point out that $\left(B_{3}\right)^{1}$ holds by the mean value theorem together with $\left(B_{3}\right)^{0}$ and $\left(B_{3}\right)^{2}$. Rewrite (5.21) as

$$
\dot{u}(t)=-a(\infty) \int_{0}^{t} g(u(\tau)) d \tau-\int_{0}^{t}(a(t-\tau)-a(\infty)) g(u(\tau)) d \tau+f(\infty)+f_{1}(t) .
$$

Since $\left|f_{1}(t)\right|$ is bounded and $f_{1} \in L_{1}[0, \infty), f_{1}(t) \rightarrow 0$, as $t \rightarrow \infty . a-a(\infty) \in L_{1}$ and $\left(\mathrm{S}_{0}\right)$ imply the second integral above tends to zero. The desired conclusion (5.22) follows immediately.

Finally we consider Case (4). We find that the present technique for treating the nonlinear equation does not yield a full generalization of the linear result (5.12), even under the additional assumption $\left(\mathrm{G}_{5}\right)$. However, a similar result can be obtained by imposing the stronger assumption $\left(a_{9}\right)$. The necessity of $\left(a_{9}\right)$ in the validity of the following result remains an open question.

TheOREM (5.6). Assume that $\left(\mathrm{a}_{1}\right)-\left(\mathrm{a}_{4}\right),\left(\mathrm{a}_{9}\right),\left(\mathrm{G}_{1}\right),\left(\mathrm{G}_{3}\right),\left(\mathrm{G}_{4}\right)$ hold for equation (5.21), where $f_{1} \in L_{1}[0, \infty)$. Then every solution of (5.21) satisfies

$$
\lim _{t \rightarrow \infty} g(u(t))=\beta=-f(\infty) / \int_{0}^{\infty} a(s) d s .
$$

Proof. Let $u(t)$ be a solution and set

$$
v(t)=u(t)-g^{-1}(\beta), \quad h(v)=g\left(v+g^{-1}(\beta)\right)-\beta .
$$

In terms of these new variables, equation (5.21) reads

$$
\dot{v}(t)=f_{1}(t)+\beta \int_{t}^{\infty} a(t-\tau) h(v(\tau)) d \tau,
$$

which is an equation of the form (5.2) considered in Theorem (5.2). Introduce the function $H(v)=\int_{0}^{v} h(z) d z$. We find by (5.24) that

$$
H(v)=G\left(v+g^{-1}(\beta)\right)-G\left(g^{-1}(\beta)\right)-\beta v .
$$

Note that $H(v)>0$ for $v \neq 0\left(h^{\prime}(v)>0\right.$ if and only if $\left.g^{\prime}(u)>0\right)$. Moreover, since $H^{\prime \prime}(v)=h^{\prime}(v)>0, H(v) \rightarrow+\infty$ as $|v| \rightarrow \infty$ on account of $\left(\mathrm{G}_{1}\right)$. For $v \geqq 1$, we have

$$
H(v)=\int_{0}^{1} h(z) d z+\int_{1}^{v} h(z) d z \geqq h(1)(v-1),
$$


so that $v \leqq(1 / h(1)) H(v)+1$. Using this, we find by $\left(\mathrm{G}_{4}\right)$ and $(5.26)$ that

$$
\begin{aligned}
|h(v)| & =\left|g\left(v+g^{-1}(\beta)\right)-\beta\right| \leqq M\left(1+G\left(v+g^{-1}(\beta)\right)\right)+|\beta| \\
& \leqq|\beta|+M\left(1+H(v)+G\left(g^{-1}(\beta)\right)+|\beta| v\right) \\
& \leqq|\beta|+M\left(1+|\beta|+G^{-1}\left(g^{-1}(\beta)\right)\right)+M(1+|\beta| / h(1)) H(v) \\
& \leqq K(1+H(v)),
\end{aligned}
$$

for some appropriate constant $K$. We apply Theorem (5.2)(ii) to (5.25) and infer that $h(v(t)) \rightarrow 0$ as $t \rightarrow \infty$ which is (5.23).

We now return to the discussion of a slightly more general equation than (5.21), namely

$$
\dot{u}(t)=-b(t)-\int_{0}^{t} a(t-\tau) g(u(\tau)) d \tau+f(t),
$$

where $b(t)$ behaves roughly like a constant. Such an equation was considered in Levin and Nohel [10]. The results were extended by Hannsgen [6]. In both [6] and [10], the following conditions on $b(t)$ were assumed:

$\left(b_{0}\right)$ There exists a function $c(t) \in C^{1}[0, \infty)$ such that

(i) $b^{2}(t) \leqq a(t) c(t)$,

(ii) $\left(b^{\prime}(t)\right)^{2} \leqq a^{\prime}(t) c^{\prime}(t)$,

(iii) $\left|b^{\prime}(t)\right| \leqq B$.

In case $b(t) \equiv b$, and $a(\infty) \neq 0$, we may take $c(t) \equiv b^{2} / a(\infty)$ a constant. Our purpose here is to show that a result similar to Theorem (5.2) holds for equation (5.27), thus generalizing the above mentioned results.

TheOREM (5.7). Assume that $\left(\mathrm{a}_{1}\right)-\left(\mathrm{a}_{4}\right),\left(\mathrm{a}_{8}\right),\left(\mathrm{G}_{1}\right),\left(\mathrm{G}_{3}\right),\left(\mathrm{G}_{4}\right),\left(\mathrm{b}_{0}\right),\left(\mathrm{F}_{0}\right),\left(\mathrm{F}_{1}\right),\left(\mathrm{F}_{2}\right)$ hold. Then every solution $u(t)$ of $(5.27)$ satisfies $\left(\mathrm{S}_{1}\right)^{0}$.

Proof. Without loss of generality, we may assume that $a(t)>0$ and $a^{\prime}(t)<0$ for all $t>0$. As in Theorem (3.2) we "complete the square" and obtain the following energy inequality:

$$
\begin{aligned}
G(u(T))-G(u(0)) \leqq & -\frac{a(T)}{2}\left(\sigma(T)+\frac{b(T)}{a(T)}\right)^{2}+\frac{b^{2}(T)}{2 a(T)}-\int_{0}^{T} \frac{b^{\prime 2}(t)}{2 a^{\prime}(t)} d t \\
& +\int_{0}^{T} \frac{a^{\prime}(t)}{2}\left(\sigma(t)+\frac{b^{\prime}(t)}{a^{\prime}(t)}\right)^{2} d t-\frac{1}{2} \int_{0}^{T} H(t) d t+K_{0} \\
& +\frac{1}{2} \int_{0}^{T} a^{\prime}(\tau)\left(\int_{T-\tau}^{T} g(u(s)) d s\right)^{2} d \tau+\int_{0}^{T}|f(t)| G(u(t)) d t,
\end{aligned}
$$

where $\sigma(t)=\int_{0}^{t} g(u(\tau)) d \tau, H(t)=\int_{0}^{t}\left(\int_{t-\tau}^{t} g(u(s)) d s\right)^{2} d a^{\prime}(\tau)$, and $K_{0}$ is some appropriate constant. An application of condition $\left(b_{0}\right)$ to the second and third terms of the right-hand side of $(5.28)$ yields $\left(\mathrm{B}_{0}\right)$, that is $G(u(t))$ is bounded. To apply our weak stability principle we need to show that $Q_{a}[g(u) ; T]$ is bounded. We note that

$$
\begin{aligned}
Q_{a}[g(u) ; T]= & \frac{a(T) \sigma^{2}(T)}{2}-\int_{0}^{T} a^{\prime}(t) \frac{\sigma^{2}(t)}{2} d t \\
& +\frac{1}{2} \int_{0}^{T} H(t) d t-\frac{1}{2} \int_{0}^{T} a^{\prime}(\tau)\left(\int_{T-\tau}^{T} g(u(s)) d s\right)^{2} d \tau .
\end{aligned}
$$


The boundedness of the last two terms on the right of (5.29) follows from the energy inequality (5.28). From (5.28), we also have

$$
\begin{array}{r}
\frac{1}{2} a(T) \sigma^{2}(T)+b(T) \sigma(T) \leqq M_{1}, \\
-\int_{0}^{T}\left(\frac{a^{\prime}(t) \sigma^{2}(t)}{2}+b^{\prime}(t) \sigma(t)\right) d t \leqq M_{2} .
\end{array}
$$

Note that condition $\left(\mathrm{b}_{0}\right)$ implies that $c^{\prime}(t) \leqq 0$ or $c(t) \leqq c(0), t \geqq 0$. Thus,

$$
|b(t) \sigma(t)| \leqq c(0)|a(t) \sigma(t)|
$$

Also,

$$
\begin{aligned}
\int_{0}^{t} b^{\prime}(\tau) \sigma(\tau) d \tau & \leqq\left(\int_{0}^{t} \frac{b^{\prime 2}(\tau)}{-a^{\prime}(\tau)} d \tau\right)^{1 / 2}\left(\int_{0}^{t}-a^{\prime}(\tau) \sigma^{2}(\tau) d \tau\right)^{1 / 2} \\
& =(c(0)-c(t))^{1 / 2}\left(\int_{0}^{t}-a^{\prime}(\tau) \sigma^{2}(\tau) d \tau\right)^{1 / 2} .
\end{aligned}
$$

Using (5.32) and (5.33) in (5.30) and (5.31), we obtain, by a standard argument, that $a(t) \sigma^{2}(t)$ and $-\int_{0}^{T} a^{\prime}(\tau) \sigma^{2}(\tau) d \tau$ are bounded for all $t, T \geqq 0$. This together with (5.29) yields the boundedness of $Q_{a}[g(u) ; T]$. The uniform continuity of $g(u(t))$ follows from $\left(\mathrm{F}_{2}\right)$ and $\left(\mathrm{b}_{0}\right)$ (iii). This completes the proof.

REMARK (5.4). If we assume in addition that $\left(\mathrm{a}_{5}\right)$ holds, then we can consider more general equations with perturbing terms $f(t, u)$ under assumption $\left(\mathrm{F}_{3}\right)$. An extension of this type has been considered by Levin and Nohel [10]. We refer the reader to their work for further details.

We are now in a position to amplify the remarks concerning second order equations discussed in $\S 3$. We consider equations of the following form:

$$
\ddot{u}(t)=c g(u(t))-\int_{0}^{t} b(t-\tau) g(u(\tau)) d \tau+h(t) .
$$

Setting $a(t)=c+\int_{0}^{t} b(\tau) d \tau$, we can rewrite (5.34) as

$$
\ddot{u}(t)=c g(u(t))-\int_{0}^{t} a^{\prime}(t-\tau) g(u(\tau)) d \tau+h(t) .
$$

Integrating (5.35), we have

$$
\dot{u}(t)=-\int_{0}^{t} a(t-\tau) g(u(\tau)) d \tau+\dot{u}(0)+\int_{0}^{t} h(\tau) d \tau .
$$

Applying Theorem (5.6) to (5.36), we obtain

THEOREM (5.8). Suppose that $(-1)^{k+1} b^{k}(t) \geqq 0, k=0,1$, and $\left(\mathrm{G}_{1}\right),\left(\mathrm{G}_{3}\right),\left(\mathrm{G}_{4}\right)$ hold and that

$$
c+\int_{0}^{\infty} b(\tau) d \tau>0
$$


Furthermore assume that $h \in L_{1}[0, \infty)$ and $\int_{t}^{\infty} h(\tau) d \tau \in L_{1}[0, \infty)$. Then

(i) every solution of $(5.36)$ satisfies $\left(\mathrm{S}_{0}\right)$ and $\left(\mathrm{S}_{1}\right)^{0}$.

(ii) If, in addition, $c+\int_{0}^{t} b \in L_{1}$ then every solution of (5.36) satisfies

$$
\lim _{t \rightarrow \infty} g(u(t))=\left(u(0)+\int_{0}^{\infty} h(t) d t\right) / \int_{0}^{\infty} a(t) d t,
$$

where $a(t)=c+\int_{0}^{t} b(\tau) d \tau$.

Conclusion (i) given in Theorem (5.8) is closely related to a result in [12] for the linear equation where the connection with viscoelasticity is pointed out. This result of [12] is complemented by conclusion (ii) which shows that asymptotic stability is lost when $a(\infty)=0$. Condition (5.37) was also used by Dafermos [1] in connection with problems of linear viscoelasticity.

6. Partial differential functional equations. In this section, we give some first steps in the application of the weak stability principle in infinite dimensional spaces, a situation rather different from finite dimensional spaces. The basic problem in the theory is to obtain the weak uniform continuity. We have obtained complete results only in the linear case. We present two examples of the linear theory, one in some detail. This is followed by a discussion of a simple nonlinear case intended to isolate the difficulties.

We consider the linear equation $\left({ }^{4}\right)$

$$
u_{t}(x, t)=-\int_{0}^{t} a(t-\tau) L u(x, \tau) d \tau+f_{0}(x)+f_{1}(x, t), \quad x \in \Omega,
$$

where the basic space $\mathscr{H}=L_{2}(\Omega), \Omega$ a bounded region in $R^{n}$, together with the boundary condition $u=0$ on $\partial \Omega$. $L$ is a symmetric strongly elliptic operator of the form

$$
L u=\sum_{|\alpha| \leqq m:|\beta| \leqq m}(-1)^{|\alpha|} D^{\alpha}\left(a^{\alpha \beta} D^{\beta} u\right),
$$

where the coefficients $a^{\alpha \beta}=a^{\beta \alpha}$ are smooth functions which depend only on $x$. The notation here is the standard one: $\alpha=\left(\alpha_{1}, \ldots, \alpha_{m}\right)$ with the $\alpha_{i}$ 's nonnegative integers, $|\alpha|=\alpha_{1}+\cdots+\alpha_{m}$ and

$$
D^{\alpha} u=\left(\partial / \partial x_{1}\right)^{\alpha_{1}} \cdots\left(\partial / \partial x_{m}\right)^{\alpha} u \text {. }
$$

In order to obtain certain a priori estimates on the solution, we require some well-known results in partial differential equations. First, we require that solutions should be in $H_{m}^{0}$ which is the completion of $C_{0}^{\infty}(\Omega)$ under the norm

$$
\|u\|_{m}^{2}=\sum_{|\alpha| \leqq m} \int_{\Omega}\left(D^{\alpha} u\right)^{2} d x
$$

$\left(^{4}\right)$ The results of $\$ 4$ permit an obvious extension to systems of equations. 
(Thus, we are imposing 'Dirichlet' boundary conditions.) To avoid technical statements of hypothesis, we assume from the outset that $a^{\alpha \beta} \in C^{\infty}(\bar{\Omega}), \partial \Omega$ is $C^{\infty}$ and $f \in C_{0}^{\infty}(\Omega)$.

Equation (6.1) is of the form (3.1) for which results in $\S 3$ are applicable. $A(t)$ is simply $a(t)$ times the identity, therefore it defines a one parameter family of bounded linear operators on $L_{2}(\Omega)$, and $g$ is the differential operator $L$ which is accordingly unbounded with domain dense in $L_{2}(\Omega)$. On the space $H_{m}^{0}$ there is the bilinear functional $B(u, v)$ defined by

$$
B(u, v)=\int_{\Omega}(L u) v d x=\sum_{|\alpha| \leqq m ;|\beta| \leqq m} \int_{\Omega} D^{\alpha} v a^{\alpha \beta} D^{\beta} u d x .
$$

This definition is made first for $u$ and $v$ in $C_{0}^{\infty}(\Omega)$ and then by completion for $u, v \in H_{m}^{0}$. It is well known from results on elliptic partial differential operators that

LEMMA (6.1). For all $u, v \in H_{m}^{0}$,

(i) $|B(u, v)| \leqq K\|u\|_{m}\|v\|_{m}$;

(ii) (Gårding's inequality) $B(u, u) \geqq c_{1}\|u\|_{m}^{2}-c_{0}\|u\|_{0}^{2}$, where $c_{0}, c_{1}$ are positive constants and \|\|$_{0}$ denotes the $L_{2}$ norm.

We now make a special assumption on the operator $L$ :

(M) the constant $c_{0}$ in Gårding's inequality can be taken as zero.

(This is precisely the condition that the generalized Dirichlet problem for $L u=f$ should have a solution. For further comment see Remark (6.1) following the proof of the theorem.) We can now state the main result of this section.

THEOREM (6.1). Let $\{a(t): t \geqq 0\}$ define a strongly positive kernel. Suppose that $a(\infty)$ exists and is positive and that assumption $\left(\mathrm{a}_{7}\right)$ is satisfied. Then if

$$
\left\|f_{1}(\cdot, t)\right\|_{m} \in L_{1}(0, \infty)
$$

and

$$
\left\|f_{1, t}(\cdot, t)\right\|_{m} \in L_{1}(0, \infty)
$$

any solution of (6.1) satisfies

$$
\lim _{t \rightarrow \infty}\|u(\cdot, t)\|_{m}=0
$$

Proof. We will use the weak stability principle, Theorem (2.1). Let us assume for the moment that we have established the necessary conditions to apply Theorem (2.1). Then the conclusion will be that $L u$ converges weakly to zero in $L_{2}(\Omega)$. We will show that this implies the desired conclusion (6.7).

The weak convergence of $L u$ implies first that

$$
\|L u\|_{0} \leqq K_{0}, \quad K_{0} \text { constant. }
$$


As part of the proof to follow we will also show that $\|u\|_{0}$ is bounded. Then it follows from a priori inequalities for strongly elliptic equations (see [2, Theorem 18.1]) and (6.8) that

$$
\|u\|_{2 m} \leqq K_{1}, \quad K_{1} \text { constant. }
$$

Here $\|\cdot\|_{2 m}$ is formed in the same way as $\|\cdot\|_{m}$ in (6.3). Suppose now that $\|u(t)\|_{m}$ does not tend to zero. Then there exists an $\varepsilon>0$ and a sequence $t_{n} \rightarrow \infty$ such that $\left\|u\left(t_{n}\right)\right\| \geqq \varepsilon$. Now by (6.9), we know that the set $\left\{u\left(t_{n}\right)\right\}$ is bounded in $H_{2 m}^{0}$ (the completion of $C_{0}^{\infty}(\Omega)$ under $\left.\|\cdot\|_{2 m}\right)$. It is a standard theorem in partial differential equations that the embedding $H_{k}^{0} \rightarrow H_{j}^{0}$ is compact if $k>j$. Thus the set $\left\{u\left(t_{n}\right)\right\}$ is compact in $H_{2 m-j}^{0}$ for any $j, 1 \leqq j \leqq 2 m$, and accordingly a subsequence $u\left(t_{n_{k}}\right)$ converges to $\bar{u} \in H_{m}^{0}$. But this subsequence must converge to zero since, for each $v \in H_{m}^{0}$,

$$
0=\lim _{k \rightarrow \infty}\left(L u\left(t_{n_{k}}\right), v\right)=\lim _{k \rightarrow \infty} B\left(u\left(t_{n_{k}}\right), v\right)
$$

Moreover, by Lemma $(6.1)(\mathrm{i}), B(u, v)$ is continuous in $H_{m}^{0}$ for a fixed $v$. Thus $B(\bar{u}, v)=0$ for all $v \in H_{m}^{0}$, hence $\bar{u}=0$. This contradicts the assumption $\left\|u\left(t_{n}\right)\right\|_{m}$ $\geqq \varepsilon>0$.

We now turn to a proof that the weak stability principle is applicable. Following Remark (3.3), we define $G(u)$ by

$$
G(u)=\frac{1}{2}(u, L u)=\frac{1}{2} B(u, u) .
$$

It follows immediately from Gårding's inequality, under assumption (M), that assumption $\left(\mathrm{G}_{1}\right)$ of $\S 3$ is satisfied. Moreover we have $(d / d t) G(u)=\left(u_{t}, L u\right)$. Also, for any $u, v \in H_{m}^{0}$ we have

$$
|B(u, v)|^{2} \leqq K^{2} B(u, u)\|v\|_{m}^{2}, \quad K \text { constant. }
$$

Thus, we have

$$
\begin{aligned}
|(L u, f)| & =|B(u, f)| \leqq K(B(u, u))^{1 / 2}\|f\|_{m} \\
& =2^{1 / 2} K(G(u))^{1 / 2}\|f\|_{m} .
\end{aligned}
$$

Note that

$$
2 G(u) \leqq 1+G^{2}(u) \leqq(1+G(u))^{2},
$$

which, together with (6.12), implies assumption $\left(F_{0}\right)$ in $\S 3$. Thus, by Theorem (3.2), we deduce that $G(u)$ and $Q_{A}[L u: T]$ are bounded. From the boundedness of $B(u, u)$ and Gårding's inequality follows both the boundedness $\|u\|_{0}$ used above and the weak boundedness of $L u$. It remains to discuss the requirement of weak uniform continuity. Differentiating (6.1), we obtain

$$
u_{t t}(x, t)=-\int_{0}^{t} a(t-\tau) L u_{\tau}(x, \tau) d \tau+\tilde{f}(x, t),
$$


where $\tilde{f}(x, t)=-a(t) L u(x, 0)+\tilde{f}_{1, t}(x, t)$. With the assumption (6.6), we can consider equation (6.13) in the same form as equation (6.1), where $\tilde{f}(x, t)=\tilde{f}_{0}(x)$ $+\tilde{f}_{1}(x, t), \tilde{f}_{0}(x)=-a(\infty) L u(x, 0)$ and $\tilde{f}_{1}(x, t)=-(a(t)-a(\infty)) L u(x, 0)+\tilde{f}_{1, t}(x, t)$. $\tilde{f}_{0}$ and $\tilde{f}_{1}$ satisfy the same conditions as $f_{0}(x)$ and $f_{1}(x, t)$. Thus, an application of Theorem (3.2) yields the weak boundedness of $L u_{t}$, hence the weak uniform continuity of $L u$. This completes the proof.

Corollary (6.1). Let $\{a(t): t \geqq 0\}$ define a positive kernel. Suppose that $\|a(t)\|$ and $\|f(\cdot, t)\|_{m},\left\|f^{\prime}(\cdot, t)\right\|_{m} \in L_{1}(0, \infty)$. Then every solution of

$$
u_{t}(x, t)=-\int_{0}^{t} a(t-\tau) L u(x, t) d \tau+f(x, t)
$$

satisfies (6.7).

REMARK (6.1). Something like hypothesis (M) is necessary if we are to obtain asymptotic stability as the following example shows. Consider the equation

$$
u_{t}(x, t)=\int_{0}^{t} e^{-\alpha(t-\tau)}\left(u_{x x}(x, \tau)+\lambda u(x, \tau)\right) d \tau,
$$

with $u(0)=u(1)=0$. Here, the energy function $G(u)$ is determined by

$$
B(u, u)=\int_{0}^{1}\left(u_{x}^{2}-\lambda u^{2}\right) d x
$$

so $c_{0}=c_{1}=1$ in Gårding's inequality. It is not hard to see that if $\lambda$ is large enough there will be solutions of (6.14) of the form $u(x, t)=T(t) \sin \pi x$ in which $T$ grows exponentially with $t$. We remark that (M) is always satisfied if $L$ is homogeneous of degree $2 m$ with constant coefficients.

We now return to equation (6.1) and consider the situation when $f_{0}(x) \not \equiv 0$ and $a(t) \in L_{1}(0, \infty)$, a case also considered in the one dimensional equation. Here also we show that in some cases asymptotic stability cannot be expected.

We observe that the differential operator $L$ has a bounded inverse if we restrict the range of $L^{-1} u$ to lie in $H_{m}^{0}$. This observation is simply the statement that the generalized Dirichlet boundary value problem, $L u=f$, has a solution in $H_{m}^{0}$ for every $f \in L_{2}(\Omega)$.

THEOREM (6.2). Suppose that a(t) satisfies assumptions $\left(\mathrm{a}_{1}\right)-\left(\mathrm{a}_{4}\right)$ in $\S 4$ and that $a(t) \in L_{1}(0, \infty)$ and $\int_{t}^{\infty} a(\tau) d \tau \in L_{1}(0, \infty)$. Furthermore, we assume that hypothesis (M) holds for L. Let $u$ be a solution of

$$
u_{t}(x, t)=-\int_{0}^{t} a(t-\tau) L u(x, \tau) d \tau+f_{0}(x)+f_{1}(x, t),
$$

where $f_{0} \in L_{2}(\Omega)$ and $f_{1}$ satisfies (6.5) and (6.6). Then $u$ satisfies the following:

$$
\lim _{t \rightarrow \infty}\|u(\cdot, t)-\bar{u}(\cdot)\|_{m}=0
$$


where $\bar{u}(x)$ is the (unique) solution to the generalized Dirichlet problem $L u=\lambda f_{0}$, $u \in H_{m}^{0}$, with $\lambda=\int_{0}^{\infty} a(t) d t$.

Proof. We can rewrite (6.15) as

$$
u_{t}(x, t)=-\int_{0}^{t} a(t-\tau)(L u(x, \tau)-L \bar{u}(x)) d \tau+\left(\int_{t}^{\infty} a(\tau) d \tau\right) f_{0}(x)+f_{1}(x, t) .
$$

Let $v(x, t)=u(x, t)-\bar{u}(x)$. We see from (6.17) that

$$
v_{t}(x, t)=-\int_{0}^{t} a(t-\tau) L v(x, \tau) d \tau+\left(\int_{t}^{\infty} a(\tau) d \tau\right) f_{0}(x)+f_{1}(x, t),
$$

which is of the form (6.1). The desired result follows from Corollary (6.1).

Results given in Theorems (6.1) and (6.2) allow us to treat some second order equations in much the same way as in the one dimensional case. Consider

$$
u_{t t}(x, t)=-a(0) L u(x, t)-\int_{0}^{t} a(t-\tau) L u(x, \tau) d \tau+f(x, t),
$$

which can be transformed into equation (6.1) by a simple integration. If we apply Theorem (6.1), we have the following result:

THEOREM (6.3). Suppose that $\left(\mathrm{a}_{1}\right)-\left(\mathrm{a}_{4}\right)$ of $\S 4$ are satisfied for $a(t)$. Suppose further that the operator L satisfies hypothesis $(\mathrm{M})$ and $f$ satisfies $(6.5)$ and

$$
\left\|\int_{t}^{\infty} f(\cdot, \tau) d \tau\right\|_{m} \in L_{1}(0, \infty)
$$

Then every solution of (6.18) satisfies (6.7).

REMARK (6.2). Dafermos [1] proves a theorem which is similar to Theorem (6.3) in the case where $\Omega$ is a bounded interval in $\boldsymbol{R}^{1}$. His proof is based upon the construction of a certain Lyapunov function, a technique used by Levin [8].

We close our discussion with some remarks concerning a nonlinear problem. Consider the equation

$$
u_{t}(x, t)=\int_{0}^{t} a(t-\tau) \frac{\partial}{\partial x} \sigma\left(u_{x}\right) d \tau, \quad u(0)=u(1)=0
$$

where the underlying space is $\mathscr{H}=L_{2}(0,1)$. We assume as before that $a(t)$ satisfies conditions $\left(a_{1}\right)-\left(a_{4}\right)$ in $\S 4$ and hence $a(t) I$ defines a strongly positive kernel on $L_{2}(0,1)$. To find a suitable energy function $G(u)$, we cannot use the general idea given in $\S 3$ but we can use the special form of (6.19). Define the function $G(u)$ on $L_{2}(0,1) \cap C^{1}(0,1)$ by

$$
G(u)=\int_{0}^{1}\left(\int_{0}^{u_{x}} \sigma(\xi) d \xi\right) d x
$$


Then we have

$$
\frac{d}{d t} G(u)=\int_{0}^{1} \sigma\left(u_{x}\right) u_{x t} d x=\left(\sigma\left(u_{x}\right), u_{x t}\right)
$$

If we impose the further condition

$$
\sigma^{\prime}(\xi) \geqq m>0, \quad \sigma(0)=0,
$$

then we have

$$
G(u) \geqq \frac{1}{2} m \int_{0}^{1} u_{x}^{2} d x
$$

Recall that for functions vanishing at 0 and 1 we always have

$$
\int_{0}^{1} u_{x}^{2} d x \geqq c \int_{0}^{1} u^{2} d x, \quad c>0 .
$$

Hence (6.23) and (6.24) together imply that $G$, as defined by (6.20), satisfies hypotheses $\left(\mathrm{G}_{0}\right)$ and $\left(\mathrm{G}_{1}\right)$ of $\S 3$. Theorem (3.1) implies that $G(u)$ and $Q_{\tilde{A}}[g(u) ; T]$ are bounded and, in particular, $\|u\|_{1}$ and $\|u\|_{0}$ are also bounded for all $t \geqq 0$. To obtain the second part of Theorem (3.1), we observe that

$$
(g(u), v)=\int_{0}^{1} \frac{\partial}{\partial x} \sigma\left(u_{x}\right) v d x=\int_{0}^{1} \sigma\left(u_{x}\right) v_{x} d x,
$$

for all $v \in C_{0}^{\infty}[0,1]$. Since $C_{0}^{\infty}[0,1]$ is dense in $\mathscr{H}$, weak boundedness of $g(u)$ will follow if we can show that the last integral in (6.25) is bounded for each fixed $v$. We come now to the first difficulty with nonlinear problems, namely, the estimate (6.23) does not apply to (6.25) so as to imply weak boundedness of $g(u)$. More precisely, since $\sigma$ is not a linear function, we cannot use Schwarz inequality as in the previous case with linear equations. Thus, we have to make further assumptions on $\sigma$. We assume that

$$
|\sigma(\xi)| \leqq m|\xi|+K|\xi|^{r}, \quad 0<r<2 .
$$

Using (6.26), we may estimate $(g(u), v)$ in $(6.25)$ by Hölder's inequality as follows:

$$
|(g(u), v)| \leqq K_{1} \int_{0}^{1} u_{x}^{2} d x+K_{2}\left(\int_{0}^{1} u_{x}^{2} d x\right)^{r / 2},
$$

where $K_{1}, K_{2}$ are positive constants depending on $v \in C_{0}^{\infty}[0,1]$. Thus for the type of nonlinearity (6.26), we obtain weak boundedness of solutions of (6.19). Other algebraic rate of growth conditions may be imposed on $\sigma$ instead of (6.26); for example, let $\sigma(\xi)=|\xi|^{\alpha} \operatorname{sgn} \xi, \alpha>0$. Then, the energy functional $G$ is given by

$$
G(u)=\frac{1}{\alpha+1} \int_{0}^{1}\left|u_{x}\right|^{\alpha+1} d x .
$$


Thus, we can apply Hölder's inequality to obtain

$$
|(g(u), v)| \leqq C_{0}\left(\int_{0}^{1}\left|u_{x}\right|^{\alpha+1} d x\right)^{1+1 / \alpha}
$$

where $C_{0}$ is a constant depending on $v$.

To obtain weak stability of solutions of equation (6.19), we need to verify the weak uniform continuity of $g(u)$, namely the uniform continuity of

$$
\int_{0}^{1} \sigma\left(u_{x}(x, t)\right) v_{x}(x) d x
$$

for all $v \in C_{0}^{\infty}[0,1]$. If this condition is satisfied, then we obtain from Theorem (3.1) that $(\partial / \partial x) \sigma\left(u_{x}\right)$ converges weakly to zero. Note that the weak convergence of $(\partial / \partial x) \sigma\left(u_{x}\right)$ implies the boundedness of

$$
\int_{0}^{1}\left(\frac{\partial}{\partial x} \sigma\left(u_{x}\right)\right)^{2} d x=\int_{0}^{1} \sigma^{\prime}\left(u_{x}\right)^{2} u_{x x} d x
$$

hence, by (6.22), the boundedness of $\int_{0}^{1} u_{x x}^{2} d x$. Since the embedding of $H_{2}^{0}$ into $H_{1}^{0}$ is compact, we would deduce, as in the proof of Theorem (6.1), that

$$
\int_{0}^{1} u_{x}^{2}(x, t) d x \rightarrow 0 \text { as } t \rightarrow \infty
$$

Unfortunately, we are unable to prove the weak uniform continuity of solutions of (6.19). The device of differentiating the equation, as we did for the linear equation (6.1), of course fails for the nonlinear case.

\section{REFERENCES}

1. C. M. Dafermos, An abstract Volterra equation with applications to linear viscoelasticity, J. Differential Equations 7 (1970), 554-569. MR 41 \#4305.

2. A. Friedman, Partial differential equations of parabolic type, Prentice-Hall, Englewood Cliffs, N. J., 1964. MR 31 \#6062.

3. M. E. Gurtin and I. Herrera, On dissipation inequalities and linear viscoelasticity, Quart. Appl. Math. 23 (1965), 235-245. MR 32 \#6772.

4. A. Halanay, On the asymptotic behavior of the solutions of an integro-differential equation, J. Math. Anal. Appl. 10 (1965), 319-324. MR 31 \#579.

5. K. B. Hannsgen, Indirect abelian theorems and a linear Volterra equation, Trans. Amer. Math. Soc. 142 (1969), 539-555. MR 39 \#7364.

6. — On a nonlinear Volterra equation, Michigan Math. J. 16 (1969), 365-376. MR 40 \#3225.

7. E. Hille and R. S. Phillips, Functional analysis and semi-groups, rev. ed., Amer. Math. Soc. Colloq. Publ., vol. 31, Amer. Math. Soc., Providence, R. I., 1957. MR 19, 664.

8. J. J. Levin, The asymptotic behavior of the solution of a Volterra equation, Proc. Amer. Math. Soc. 14 (1963), 534-541. MR 27 \#2824.

9. J. J. Levin and J. A. Nohel, Note on a nonlinear Volterra equation, Proc. Amer. Math. Soc. 14 (1963), 924-929. MR 28 \#437.

10. - Perturbations of a nonlinear Volterra equation, Michigan Math. J. 12 (1965), 431-447. MR 32 \#336. 
11. M. Loève, Probability theory. Foundations. Random sequences, 2nd rev. ed., University Series in Higher Math., Van Nostrand, Princeton, N. J., 1960. MR 23 \#A670.

12. R. C. MacCamy, Exponential stability for a class of functional differential equations, Arch. Rational Mech. Anal. 40 (1971), 120-138.

13. H. König and J. Meixner, Lineare Systeme und lineare Transformationen, Math. Nachr. 19 (1958), 256-322. MR 21 \#7410.

14. J. A. Nohel, Qualitative behavior of solutions of nonlinear Volterra equations, Stability Problems of Solutions of Differential Equations, Proceedings of a NATO Advanced Study Institute, Oderisi, Gubbis, 1966, pp. 177-210.

15. M. M. Vainberg, Variational methods for the study of non-linear operators, GITTL, Moscow, 1956; English transl., Holden-Day, San Francisco, 1964. MR 19, 567; 31 \#638.

Department of Mathematics, Carnegie-Mellon University, Pittsburgh, Pennsylvania 15213

Current address (Wong): Department of Mathematics, University of Iowa, Iowa City Iowa 52240 